\title{
ARAÑAS CHILENAS: ESTADO ACTUAL DEL CONOCIMIENTO Y CLAVE PARA LAS FAMILIAS DE ARANEOMORPHAE
}

\section{CHILEAN SPIDERS: CURRENT STATE OF KNOWLEDGE AND KEY TO THE ARANEOMORPHAE FAMILIES}

\author{
Milenko A. Aguilera \& María E. Casanueva \\ Universidad de Concepción, Facultad de Ciencias Naturales y Oceanográficas. Departamento de Zoología. \\ Casilla 160-C, Concepción, Chile.E-mail: miaguile@udec.cl
}

\begin{abstract}
RESUMEN
La aracnofauna chilena ha sido parcialmente estudiada a través de los años, pero no en forma continua, lo que ha determinado un escaso conocimiento de los taxones presentes en nuestro país. La ausencia de textos y manuales que faciliten la identificación, y determinación taxonómica de las arañas presentes en Chile, hace aún más difícil el estudio de este grupo. Para Chile se han descrito 55 familias, de las cuales 6 pertenecen a Mygalomorphae y 49 a Araneomorphae, lo que representa aproximadamente el 50\% de las familias conocidas a nivel mundial. En este estudio se reconocen 51 familias. Es importante destacar la necesidad de continuar con estudios sistemáticos de las arañas presentes en Chile, y determinar su biología, distribución geográfica y diversidad. Se entregan antecedentes biológicos y una clave dicotómica para las familias de Araneomorphae más comunes para el país. En la clave no se han incluido las familias de araneomorfas: Amphinectidae, Clubionidae, Desidae, Synotaxidae, Tengellidae y Titanoecidae, por presentar problemas taxonómicos, como tampoco las familias de migalomorfas.
\end{abstract}

Palabras Claves: Arañas, sistemática, clave, antecedentes biológicos, Chile.

\section{ABSTRACT}

The studies of the chilean arachnofauna have been scarce during the past years and there is a lack of texts and identification keys to help taxonomical and biological studies in Chile. Therefore the knowledgment is still poor. A total of 55 families are reported for Chile, 6 belonging to Mygalomorphae and 49 to Araneomorphae, which represents around the $50 \%$ of the world known families. Only 51 are reconized for Chile in this study. It is important to develop new systematic studies of the spiders present in Chile to determine their biology, geographical distribution and diversity. Biological information and a dicotomic key for the more common families of Araneomorphae are given. Mygalomorph families and the araneomorph families Amphinectidae, Clubionidae, Desidae, Synotaxidae, Tengellidae y Titanoecidae and are not included in the present key due to taxonomical problems of the later ones.

KEYwords: Spiders, systematic, key, biological information, Chile.

\section{INTRODUCCION}

Las arañas ocupan el sexto o séptimo lugar de órdenes de animales en términos de diversidad (Coddington \& Levi 1991), con alrededor de 38.000 especies válidamente descritas en 3.607 géneros y 110 familias y cuyo número real podría alcanzar la cifra de 170.000 especies (Platnick 1989, 2005). La aracnofauna de Norteamérica, oeste de Europa y Japón son las mejores estudiadas, pero de Australia y las zonas templadas del sur aún son pobremente conocidas, especialmente las arañas de las regiones tropicales (Coddington \& Levi op. cit.).

El estudio de las arañas chilenas ha tenido una larga 
trayectoria, pero no ha sido lo suficientemente continua como para tener un conocimiento acabado de los taxa presentes en el país. Es así que existen pocas revisiones de las familias presentes en Chile con métodos modernos, y una ausencia de textos actualizados con claves para las familias. Actualmente de no ser por el interés mostrado por científicos extranjeros, éste continuaría siendo un grupo parcialmente estudiado.

El conocimiento de la aracnofauna chilena fue iniciado con el abate Juan Ignacio Molina, al describir la migalomorfa Phrixotrichus scrofa (Paraphysa scrofa (Molina 1782)), y porque en su libro "Saggio sulla Storia Naturale del Chili" determina otras especies de arañas presentes en el país (Molina 1782). En 1849 Nicolet publica descripciones de diversas especies de arañas chilenas en el texto de Claudio Gay "Historia Física y política de Chile", siendo uno de los estudios más completos para la época, ya que menciona una buena parte de la aracnofauna del país. Otro aracnólogo destacado fue Eugéne Simon (1859), quien describió nuevas especies de material proveniente de las numerosas expediciones realizadas al extremo sur de Chile. Uno de los investigadores que se dedicó al estudio de la fauna aracnológica del sur de Chile, principalmente de Aysén y Magallanes, fue Albert Tullgren (1901). Por otro lado, Porter $(1917,1918,1930)$ realizó diversos trabajos en generalidades de arañas y una revisión de la especie Aranea scopha. Ya en la década de los 20, Cándido de Mello-Leitão (1926; 1943)publicó varios trabajos en revistas científicas nacionales. Aproximadamente en 1950, Zapfe retoma el estudio de las arañas en Chile y elabora una clave (1959), determina la distribución ecológica de Araneae (1961a), la distribución altitudinal de arañas (1961b), la biogeografía de arañas chilenas (1961c), la presencia de arañas tropicales en el país (1961d), las familias presentes en el país, como Palpimanidae, Filistatidae, y Migidae (1961e, 1961f, 1961g) y varios otros trabajos descriptivos (1979). Por otro lado, Archer (1963) realiza un catálogo de las arañas chilenas de las familias de la división Metarachnae. Tomás Cekalovic (1976) publicó el catálogo de arañas de Magallanes. Casanueva (1980) realiza un estudio biológico y taxonómico de los licósidos de Chile, proporcionando claves para el reconocimiento de las distintas especies y la descripción de especies nuevas y/o endémicas. Legendre \& Calderón (1984) dieron a conocer la sistemática de las migalomorfas presentes en Chile. La única clave para familias de arañas presentes en el país es la realizada por Zapfe (1959), sin embargo su actualización es necesaria debido a que los taxa de arañas han sido modificados (Kaston 1978, Raven 1985, Platnick 1989, 2005), y porque en muchos casos las especies chilenas han sido reubicadas en otros géneros o familias (Forster \& Platnick 1984, Ramírez 1995, 1997, Ramírez et al. 1997, Ramírez et al. 2001). Por otro lado, la mayoría de las claves actualmente existentes son para géneros y familias de Norteamérica, Europa y, lo más cercano, Argentina, pero no incluyen muchas de las familias presentes en el país y además mencionan otras que no lo están.

Entre otros autores que han estudiado la aracnofauna chilena es posible destacar a Levi (1967), quien elabora un catálogo y clave de la familia Theridiidae en Chile; Galiano (1980) con el catálogo de los especímenes típicos de Salticidae, donde incluye varias especies chilenas y además realiza la revisión de varios géneros presentes en el país (1985, 1988); Raven (1985) quien hace un análisis cladístico y sistemático de las Mygalomorphae incluyendo especies presentes en Chile; Forster \& Platnick (1984, 1985) revisan las superfamilias Palpimanoidea y Dysderoidea; Platnick \& Forster $(1986,1987,1989)$ realizan descripciones de nuevos géneros y revisan la familia Anapidae; Platnick \& Shabad (1993), hace una revisión de las arañas piratas de Chile (Mimetidae); Goloboff(1994, 1995) menciona las migoideas chilenas y realiza una revisión de la familia Nemesiidae; Ramírez (1995, 1997, 2003) hace la revisión y filogenia de varios géneros de Anyphaenidae.

Entre las arañas presentes en Chile, especial interés han despertado las especies de los géneros Latrodectus y Loxosceles, cuyas mordeduras provocan graves lesiones en el ser humano. Esto ha determinado un mayor número de estudios para cuantificar los efectos del veneno de estas arañas en el hombre y en otros animales; entre estos se puede destacar estudios sobre la sintomatología del aracnoidismo (Donoso 1948, Gajardo-Tobar 1963); el latrodectismo y su tratamiento (Artaza et al. 1982, Artaza et. al. 1984, Gómez et al. 1986, 1987, Scherone 1959); el loxoscelismo (Barrio \& IbarraGrasso 1966, Schenone et al. 1975); Loxosceles sp. aspectos biológicos (Schenone \& Lentoja 1975) y recientemente, las especies de arañas peligrosas en Chile (Cannals et al. 2004). 
Por otra parte las arañas son consideradas de importancia en el equilibrio ecológico de poblaciones de invertebrados y por constituir elementos comunes dentro de ellas (Flórez 1999). Además, son uno de los grupos entomófagos más abundantes en la naturaleza (Moulder \& Riechle 1972, Nyffeler et al. 1994).

Todos los antecedentes anteriormente mencionados permiten establecer la importancia de realizar estudios tendientes a determinar un acabado conocimiento de la fauna de arañas presentes en nuestro país, como en el resto del mundo. Por lo cual en el presente trabajo se propone una clave para las familias más frecuentes en el país y se entregan antecedentes biológicos de ellas.

\section{MATERIALES Y METODOS}

En este estudio inicialmente se realizó una búsqueda bibliográfica y consulta de especialistas para determinar los posibles taxa de araneae presentes en Chile. Según la distribución geográfica de las especies dada en el catálogo mundial de arañas (Platnick 2005), se han considerado como géneros probablemente presentes en el país aquellos que presentan distribución considerada como cosmopolita y neotropical.

Luego, se hace una revisión del material de arañas depositado en las siguientes instituciones: Museo de Zoología de la Universidad de Concepción (MZUC), Colección del Laboratorio de Entomología y Ecología de la Universidad de La Serena (LEULS), Colección del Museo Nacional de Historia Natural (MNS), Colección particular de Raúl Calderón, Colección del Laboratorio de Aracnología de la Universidad de Concepción.

Una vez determinadas las familias presentes en el país se obtienen los caracteres más relevantes de cada una de ellas según los trabajos de Forster \& Platnick 1984 (Mecysmaucheniidae); Forster \& Platnick 1985 (Orsolobidae); Forster, Platnick \& Coddington 1990 (Synotaxidae); Forster, Platnick \& Gray 1987 (Austrochilidae); Huber 2000 (Pholcidae); Lehtinen 1967 (Titanoecidae); Platnick 1984, 1985, 1990 (Trochanteriidae); Platnick \& Forster 1986 (Micropholcommatidae); Platnick \& Forster 1987 (Malkaridae); Platnick, Grismado \& Ramírez 1999 (Palpimanidae); Zapfe 1955 (Austrochilidae); Roth 1993 (que incluyen la mayoría de las familias), y además observaciones personales realizadas por los autores y consultas a especialistas.

La confección de la clave dicotómica se hace sobre la base de la clave para familias de Norteamérica propuesta por Kaston (1978), de la clave para familias y géneros propuesta por Roth (1993) y la clave para familias de Argentina propuesta por Ramírez (1999). Los caracteres morfológicos utilizados en la clave corresponden a los comúnmente usados como parámetros de valor sistemático. La nomenclatura de la morfología utilizada se ha extraído principalmente de Comstock (1975); Kaston (1978); Casanueva (1980) y Roth (1993). Todas las medidas están en milímetros en ejemplares adultos. La longitud del cuerpo se mide desde el borde anterior del cefalotórax hasta el extremo posterior del abdomen y no se incluyen las patas ni quelíceros.

\section{RESULTADOS}

De las 55 familias presentes en el país 6 pertenecen a Mygalomorphae y 49 a Araneomorphae (Tabla I), de este último infraorden sólo 43 han sido incluidas en la presente clave.

No se han incluido las familias Amphinectidae, Clubionidae, Desidae, Synotaxidae, Tengellidae y Titanoecidae por presentar problemas en la definición de sus caracteres diagnósticos, en los géneros y/o especies incluidos en ellas o por problemas en la relación de parentesco; por ejemplo, se ha mencionado que los Amphinectidae, Desidae y Titanoecidae estarían emparentados con los Amaurobiidae y, por otra parte, los Synotaxidae con Theridiidae (Forster et al. 1990, Lehtinen 1967, Leech 1972 fide Roth 1993), por lo cual es complejo realizar una clara diferenciación morfológica entre ellas. Además, algunas de estas familias (v. gr. Clubionidae, Tengellidae) presentan una o dos especies y/o géneros citadas para el país, pero el material tipo se reporta como perdido (Ramírez com. pers., Wolff 1977); sin embargo, sí se incluyen sus antecedentes biológicos y distribución geográfica. Las familias pertenecientes al Infraorden Mygalomorphae, no se han incluido en esta clave, debido a que han sido consideradas por Goloboff (1994, 1995), Goloboff \& Platnick (1987) y Raven (1985). 
Gayana 69(2), 2005

\section{CLAVE PARA FAMILIAS DE ARAÑAS ARANEOMORPHAE MAS COMUNES EN CHILE \\ (Modificada de Kaston, 1978 y Roth, 1993)}

1.a Quelíceros paraxiales, movimiento del colmillo en el plano vertical (Grupo Orthognatha) (Fig. 1). Con dos pares de pulmones en libro (o filotráqueas) (Fig. 2).

Infraorden Mygalomorphae

1.b Quelíceros diaxiales, colmillo muy articulado tanto que puede ser movido en un plano transversal (Grupo Labidognatha) (Fig. 3). Con dos pares de pulmones en libro o más comúnmente con un solo par (o filotráqueas). Espiráculo traqueal simple (tráqueas verdaderas) y de posición media o con un par de espiráculos a los lados del surco epigástrico y otro en la base de las hilanderas anteriores.

Infraorden Araneomorphae

.2

(1.b) 2.a Con cribelo frente a las hilanderas (Fig. 4) y un calamistro en el metatarso IV, variando desde sólo unas pocas cerdas a una hilera completa a través de la longitud del metatarso (Fig. 5).....

2.b Sin cribelo ni calamistro.

(2.a) 3.a Dos espiráculos traqueales unidos por un pliegue. Cribelo entero (Fig. 6). Clípeo prolongado en un vértice sobre los quelíceros.

3.b Un espiráculo traqueal que en algunos casos puede ser difícil de ver.

Familia Austrochilidae

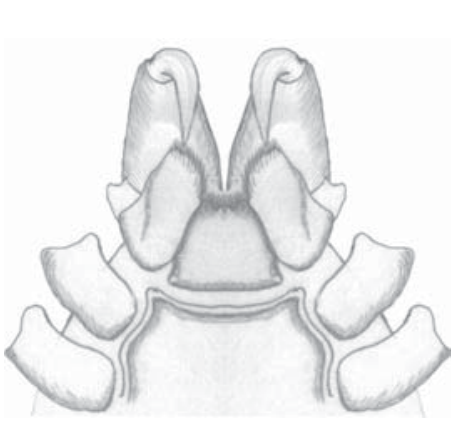

Figura 1. Theraphosidae, quelíceros paraxiales.

Figure 1. Theraphosidae, paraxial chelicerae.

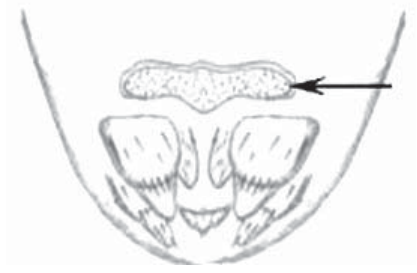

FiguRA 4. Uloboridae, hilanderas y cribelo.

FiguRE 4. Uloboridae, spinnerets and cribellum.

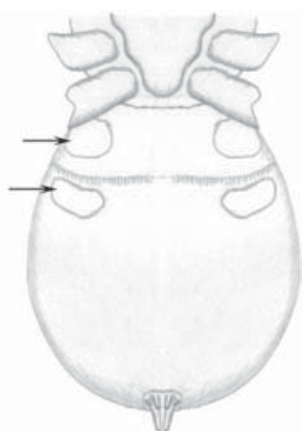

FIgURA 2. Theraphosidae, dos pares de pulmones.

FIgURE 2. Theraphosidae, two pairs of lungs.

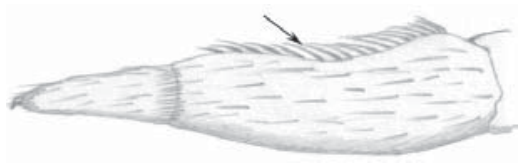

FIgURA 5. Dictynidae, metatarso con calamistro.

FIGURE 5. Dictynidae, metatarsus with calamistrum.

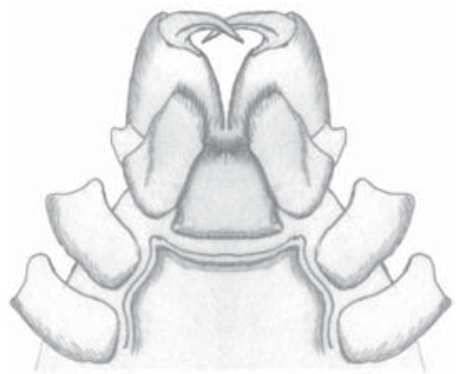

Figura 3. Araneidae, quelíceros diaxiales.

Figure 3. Araneidae, diaxial chelicerae.

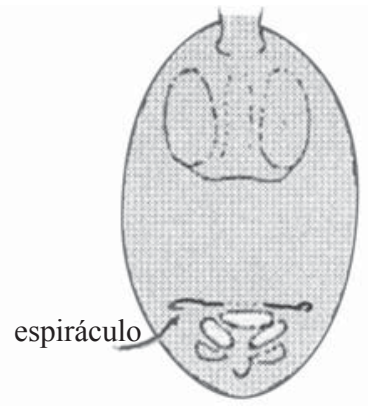

FiguRA 6. Austrochilidae, espiráculos traqueales y cribelo. (Ex.: Ramírez 1999).

Figure 6. Austrochilidae, tracheal spiracles and cribellum. (Ex.: Ramírez 1999). 
(3.b) 4.a Tubérculo anal grande y prominente igual o mayor tamaño que las hilanderas, rodeado de una fila de largos pelos (Fig. 7). Ojos posteriores medianos triangulares o de forma irregular. Cefalotórax subcircular (Fig. 8) (pequeñas arañas de 2 a $2.5 \mathrm{~mm}$. de longitud del cuerpo).

4.b Tubérculo anal de tamaño menor a las hilanderas y sin una fila de pelos....

Familia Oecobiidae

(4.b) 5.a Quelíceros fusionados en la base y cada uno con una lamela distal en forma de diente; el colmillo y la lamela asemejan una pinza (Fig. 9). Labio fusionado al esternón. Espiráculo traqueal considerablemente retirado de las hilanderas. Calamistro corto de solo unas pocas setas.

5.b Distinto a lo anterior.

Familia Filistatidae

(5.b) 6.a Metatarso VI comprimido y anteriormente cóncavo (Fig. 10). Ojos homogéneos y oscuros dispuestos en dos filas recurvas (Fig. 11).

Familia Uloboridae

6. b Metatarso IV no comprimido ni cóncavo .7

(6.b) 7.a Cribelo aparentemente dividido. Longitud del calamistro menor que la mitad de la longitud del metatarso. Patas con numerosas macrosetas y tricobotrias tarsales, estas últimas dispuestas en una fila dorsal que se incrementa en longitud distalmente.

Familia Amaurobiidae (Desidae en parte)

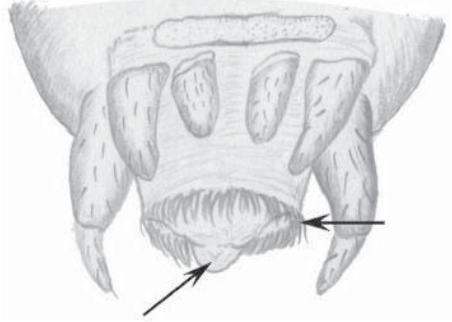

Figura 7. Oecobiidae, hilanderas, cribelo y fila de pelos en el tubérculo anal.

Figure 7. Oecobidae, spinnerets, cribellum and anal tubercle with fringe of hairs.

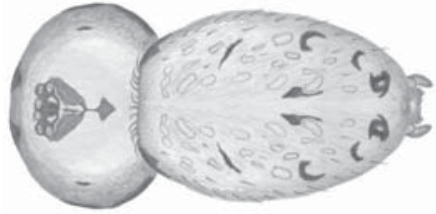

Figura 8. Oecobiidae, cefalotórax subcircular.

Figure 8. Oecobidae, subcircular carapace.

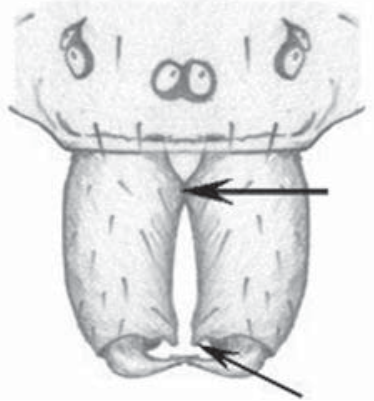

FIgURA 9. Sicariidae, quelícero vista frontal, fusionado en la base y con lamela distal

FigURE 9. Sicariidae, chelicerae, fused at the base and lamella distal.

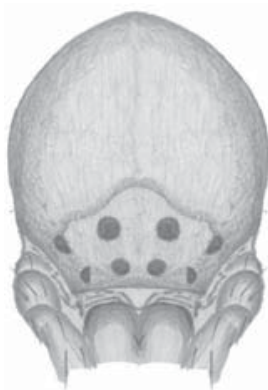

Figura 11. Uloboridae, disposición de ojos.

FIGURE 11. Uloboridae, eyes arrangement. 
Gayana 69(2), 2005

7.b Cribelo no aparentemente dividido. Calamistro casi de igual longitud que el metatarso. Sólo tibia sin una fila de macrosetas y sin espinas. Tarsos con pocas tricobotrias $(1-4)$ o ninguna. Ocho ojos heterogéneos, sólo los anteriores medianos oscuros, y la fila anterior de ojos prácticamente recta; o con seis ojos blanco perlado y ojos anteriores medianos ausentes.

Familia Dictynidae

(2.b) 8 a Sólo dos hilanderas. 8.b Seis hilanderas; leve reducción de las hilanderas posteriores sólo en Zodariidae.

(8.b) 9.a Con presencia de los siguientes caracteres combinados: quelícero originado desde un foramen en el cefalotórax y sólo dos hilanderas.

Familia Mecysmaucheniidae 9.bLas hilanderas posteriores y medianas reducidas a la fúsula; las anteriores con gran desarrollo. Ocho ojos (excepto en Hybosida spp. que pueden tener seis ojos, género no descrito para Chile). Fovea usualmente visible y simple. Surco del quelícero pobremente desarrollado o ausente; colmillo corto y grueso. Labio triangular, separado del esternón por surcos distintivos. Dos pulmones en libro y un espiráculo traqueal cercano a las hilanderas.

Familia Palpimanidae

(9.b) 10.a Presencia combinada de los siguientes caracteres: una glándula queliceral elevada en un tubérculo y depresiones circulares proximales (Figs. 36 - 39). Pulmones en libro anteriores reducidos a hojas poco elongadas o verdaderas tráqueas. (Machos típicamente con una o más apófisis en la patela palpar). (Ver descripción)

Familia Micropholcommatidae

10.b Sin la combinación de caracteres anteriores .11

(10.b) 11.a Tibia y metatarso I y II con una fila prolateral de espinas largas, y entre ellas con una fila de espinas cortas, todas las setas desarrolladas y notorias; las espinas se curvan en sus extremos e incrementan en longitud distalmente (Fig. 12).

11.b Tibia y metatarso I y II sin la fila prolateral de espinas.

Familia Mimetidae .12

(11.b) 12.a En presencia combinada de los siguientes caracteres: grandes tubérculos originados del margen del cefalotórax entre las coxas II a la IV (Fig. 34). Ausencia de una serie de escleritos ovales a cuadrados en los lados del abdomen. (Paracimbio del macho con una punta elongada ventral (Fig. 35).

Familia Malkaridae

12.b Margen del cefalotórax sin tubérculos entre las coxas. .13

(12.b) 13.a Quelíceros fusionados en la base; lamela distal siempre presente; colmillo y lamela semejan una pinza (Fig. 9). 14 13.b Quelíceros no fusionados en la base; lamela distal presente o ausente.

(13.a) 14.a Tarsos largos y flexibles con muchos seudosegmentos (Fig. 13). Espiráculo ausente. Clípeo tan alto como la longitud de los quelíceros. Palpo del macho con un prominente paracimbio prolateral. (Labio fusionado al esterno).

Familia Pholcidae

14.b Tarsos sin pseudosegmentos. Labio más largo que ancho y fusionado al esternón. Seis ojos .15

(14.b) 15.a Ojos anteriores dispuestos casi en una línea recta. Cefalotórax más largo que ancho, sólo de 2/3 con respecto al largo. Espiráculo ausente. (Tres uñas tarsales, coxas IV muy cercanas entre sí).

Familia Diguetidae 15.b Par de ojos medianos alejados hacia delante con respecto a los pares laterales. Cefalotórax más ancho que en el caso anterior. Espiráculo presente. . .16

(15.b) 16.a Cefalotórax anteriormente plano. Surco torácico notorio y longitudinal (excepto en el género Sicarius que es poco notorio) (Fig. 14). Tarso con dos uñas; pelos de los fascículos subungueales ausentes. Esternón posteriormente aguzado y en Sicarius suele ser escotado.

Familia Sicariidae 16.b Cefalotórax arqueado posteriomente (Fig. 15). Surco torácico poco notorio. Esternón truncado posteriormente. Coxas IV ampliamente separadas. Tarso con tres uñas.

Familia Scytodidae 


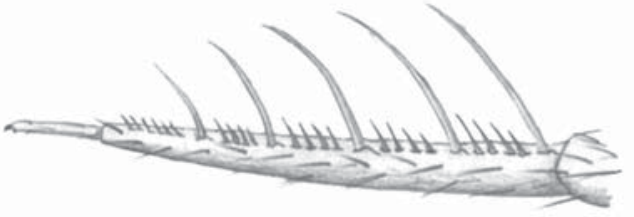

FIGURA 12. Oarces sp., espinas prolaterales en metatarso I y II.

FIGURE 12. Oarces sp., prolateral spines on metatarsi I - II.

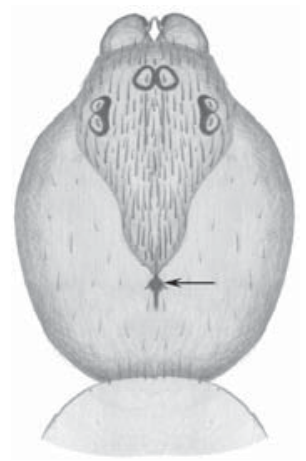

FiguRa 14. Loxosceles sp., cefalotórax anteriormente plano y fovea notoria.

FIGURE 14. Loxosceles sp., carapace with anterior region flat; thoracic furrow.

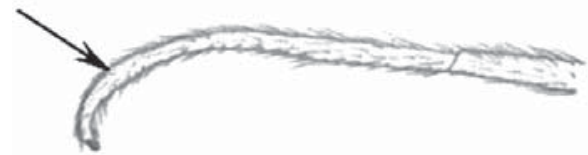

Figura 13. Pholcus sp., tarso con seudosegmentos.

FIGURE 13. Pholcus sp., tarsus with pseudosegments.

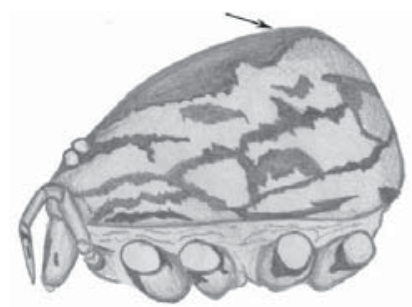

FIGURA 15. Scytodes sp., cefalotórax arqueado posteriormente.

FIGURE 15. Scytodes sp., carapace arched behind.

(13.b) 17.a Con dos, cuatro o seis ojos.

(17.a) 18.a Con dos o cuatro ojos. Hilanderas anteriores y medianas en una sola fila transversal.

18.b Con seis ojos. Hilanderas no en una fila transversal

Familia Caponiidae

(18.b) 19.a Espiráculos traqueales abiertos justo detrás del surco epigástrico.

Súper- Familia Dysderoidea.

19.b Espiráculo justo delante de las hilanderas o alrededor del medio del vientre. Clípeo mucho más bajo que alto en el área ocular. Colulo vestigial. Hilanderas anteriores muy juntas y las posteriores más largas. Longitud del cuerpo al menos de $2.5 \mathrm{~mm}$. (Pedipalpo de la hembra con una uña).

Familia Agelenidae (en parte)

(19.a) 20.a Con presencia combinada de los siguientes caracteres: un par de espiráculos posteriores detrás del surco epigástrico. Organo tarsal elevado. Uñas tarsales bipectinadas y un prominente onychium asociado con un par de cerdas propiorreceptoras en el tarso. Enditos palpales paralelos, usualmente con dientes largos en el surco del quelícero. (Pedipalpo de la hembra con uña).

Familia Orsolobidae

20.b Sin la combinación de caracteres anteriores

(20.b) 21.a Ojos medianos más grandes que los laterales; en algunas especies ojos laterales anteriores contiguos. Espiráculo traqueal poco notorio y abierto en un surco transversal. Labio tan ancho como largo. Tarso con dos uñas y con pelos espatulados. Longitud del cuerpo desde 1 a $3 \mathrm{~mm}$.

Familia Oonopidae 
21.b Distinto a lo anterior.

(21.b) 22.a Tarsos con tres uñas. Patas I, II y III dirigidas hacia delante (Fig. 16). Esternón sin extensiones laterales.

Familia Segestriidae

22.b Tarsos con dos uñas y pelos de los fascículos subungueales presentes (Fig. 17). Patas I y II dirigidas hacia delante, la III y IV dirigidas hacia atrás, como es usual. Esternón con extensión lateral entre las coxas (Fig. 18).

Familia Dysderidae

(17.b) 23.a Tarsos con dos uñas dentadas. Pelos de los fascículos subungueales presentes o ausentes. (Fig. 17)................24

23.b Tarsos con tres uñas. Pelos de los fascículos subungueales ausentes........................................................34

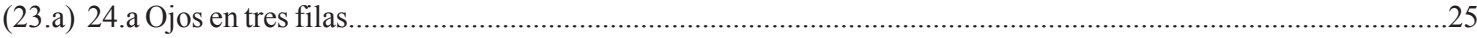

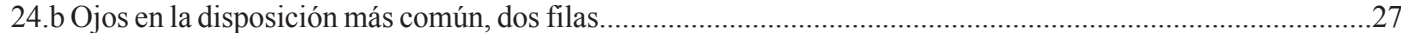

(24.a) 25.a Plano frontal del cefalotórax vertical. Ojos medianos anteriores muy grandes, dos veces más grandes que laterales anteriores; ojos de la segunda fila mucho más pequeños, a menudo diminutos; ojos de la tercera fila de tamaño medio (Fig. 19).

Familia Salticidae

25.b Plano frontal del cefalotórax no vertical. Ojos de la primera y segunda fila similares en tamaño. .26

(25.b) 26.a Primera fila con dos ojos, segunda fila con cuatro ojos y tercera fila con dos ojos; anteriores laterales más cerca de los posteriores laterales que de los anteriores medianos (Fig. 20). Surco del colmillo en el margen posterior con al menos tres dientes.

Familia Ctenidae 26.b Primera fila con cuatro ojos, segunda y tercera con dos ojos cada una; anteriores laterales más cerca de los anteriores medianos que de los posteriores laterales. El surco del colmillo en el margen posterior con dos dientes.

Familia Zoridae

(24.b) 27.a Espiráculo traqueal comúnmente desplazado desde las hilanderas hacia el surco epigástrico (Fig. 21).

27.b Espiráculo traqueal frente a las hilanderas o levemente desplazado

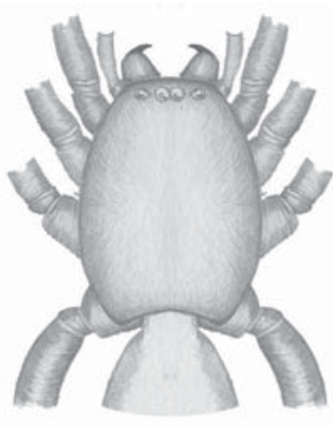

FIGURA 16. Ariadna sp., cefalotórax con patas I, II y III dirigidas hacia delante.

FIGURE 16. Ariadna sp., carapace with legs I, II and III directed forward.

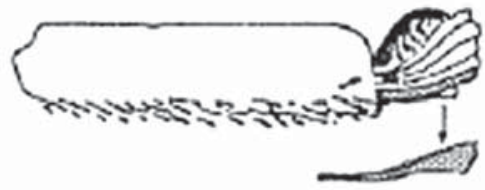

FIGURA 17. Anyphaenidae, tarso mostrando pelos de los fascículos subungueales. (Ex.: Ramírez 1999).

FIgURE 17. Anyphaenidae, tarsus with claw tuft (Ex.: Ramírez 1999).

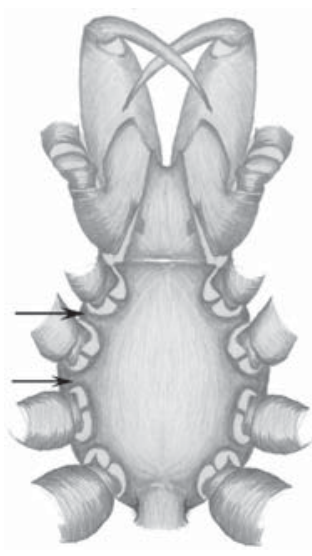

Figura 18. Vista ventral de Disdera sp. mostrando la extensión lateral entre las coxas.

Figure 18. Disdera sp., ventral view showing lateral extension between coxae. 
(27.b) 28.a Con torsión de las patas I y II, quedando la zona dorsal en posición posterior y la zona lateral anterior en posición dorsal (laterígradas) (Fig. 22).

28.b Sin torsión de ninguna pata (prógradas).

(28.a) 29.a Extremo del metatarso con una suave membrana trilobulada, permitiendo la hiperextensión del tarso.

Familia Sparassidae

29.b Extremo del metatarso esclerosado. . .30

(29.b) 30.a Pelos sobre el cuerpo simples y erguidos. Patas I y II mucho más largas y corpulentas que las III y IV (Fig.22). Mechones tarsales ausentes, o compuestos por pelos simples. Tarsos I y II no escopulados.

Familia Thomisidae 30.b Pelos sobre el cuerpo, plumosos o escamosos y no erguidos. Patas similares en longitud o pata II más larga que las restantes. Mechones tarsales compuestos de pelos espatulados. Tarsos I y II escopulados. Margen anterior del surco del quelícero con uno o dos dientes.

Familia Philodromidae

(28.b) 31.a Hilanderas anteriores cónicas, casi contiguas o juntas y no más esclerosadas que las posteriores.

. .32

31.b Hilanderas anteriores cilíndricas, largas y más esclerosadas que las posteriores y separadas por una distancia casi igual al diámetro de una de éstas (Fig. 23).

(31.a) 32.a Surco torácico reducido.Area ocular ancha, rectangular, fila posterior de ojos procurva, con ojos posteriores laterales próximos a los laterales anteriores. Artículo distal de las hilanderas laterales posteriores cónico y generalmente alargado, con fúsulas de glándulas aciniformes.

Familia Miturgidae

32.b Surco torácico notorio. En la mayoría de las especies la fila de ojos posterior casi recta y los ojos posteriores laterales son mucho más cercanos a los posteriores medianos que a los laterales anteriores. Hilanderas con fúsula de glándulas cilíndricas, grandes; al menos tres en las hilanderas posteriores medianas formando un triángulo y al menos dos en las hilanderas posteriores laterales.

Familia Corinnidae

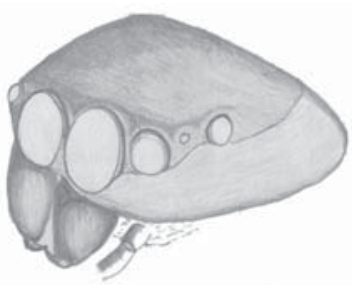

FIGURA 19. Euophrys sp., disposición de ojos.

Figure 19. Euophys sp., eyes arrangement.

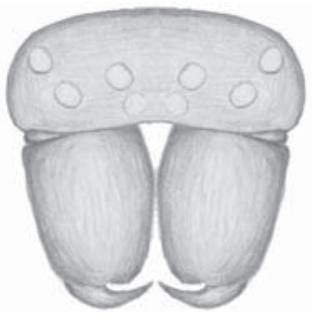

Figura 20. Ctenidae, disposición de ojos, 2-4-2.

Figure 20. Ctenidae, eyes arrangement 2-4-2.

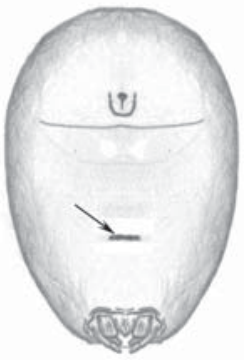

Figura 21. Abdomen de Anyphaenidae, mostrando el espiráculo traqueal.

FiguRE 21. Anyphaenidae abdomen with tracheal spiracle.

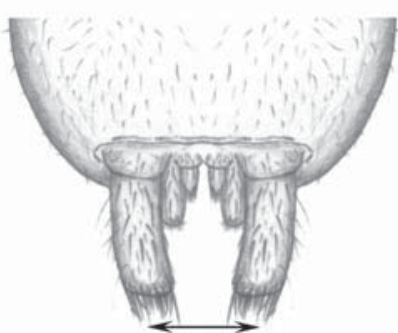

Figura 23. Gnaphosidae, hilanderas.

FIGURE 23. Gnaphosidae, spinnerets.

Figure 22. Coenypha sp., legs I and II laterigrade. 
Gayana 69(2), 2005

(31.b) 33.a Cuerpo aplastado (cefalotórax y abdomen) (Fig. 24). Extremo de las hilanderas anteriores laterales con un anillo subdistal esclerotizado; fúsulas de glándulas piriformes.

Familia Trochanteriidae 33.b Cuerpo no aplastado (cefalotórax ancho y abdomen oval u oval algo aplanado). Ojos distintamente heterogéneos, los anteriores medianos oscuros, los posteriores medianos a menudo oblicuos, ovales o triangulares. Enditos con una depresión oblicua en la cara ventral. Hilanderas anteriores laterales con gran desarrollo de fúsulas de glándulas piriformes; sin anillo esclerotizado subdistal en las hilanderas anteriores laterales.

Familia Gnaphosidae

(23.b) 34.a Seis hilanderas dispuestas en una fila que tiende a ser transversal, aun cuando pueden no estar alineadas (como en Cybaeolus spp.)

34.b Hilanderas no dispuestas en una fila transversal.

Familia Hannidae .35

(34.b) 35.a. Hilanderas posteriores levemente reducidas y pueden aparentar que sólo las anteriores están presentes. Clípeo alto, varias veces el diámetro de los ojos anteriores. Enditos palpales sin serrula.

35.b Hilanderas posteriores no reducidas.....

Familia Zodariidae

36.a Grupo de ojos formando un hexágono; fila posterior procurva y la anterior recurva. Clípeo alto. Abdomen aguzado en la parte posterior. Patas con prominentes espinas. Probablemente no esté presente en el país (ver descripción).

Familia Oxyopidae

36.b Distinto a lo anterior.

(36.b) 37.a Tarso IV, en la mayoría de las especies con una fila ventral de 6 a 10 pelos fuertes, serrados, formando un peine, en un 1/6 de su longitud. Peine del tarso IV más reducido en machos...................................................3

37.b Tarso IV sin la fila ventral de pelos serrados.

(37.a) 38.a. Pelos del peine no más largos que los pelos del lado dorsal del tarso IV. Margen del surco del colmillo del quelícero con dientes.

Familia Nesticidae 38.b Labio no rebordeado (pocas excepciones). Pelos del peine más largos que los pelos del lado dorsal del tarso IV. Margen del surco del colmillo del quelícero sin dientes en la mayoría de las especies (pocas excepciones).

\section{Familia Theridiidae}

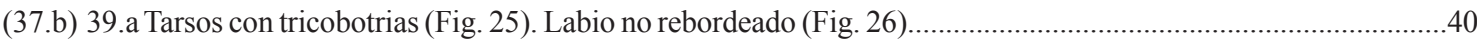

39.b Tarsos sin tricobotrias. Labio rebordeado...

(39.a) 40.a Tarsos con una fila simple de tricobotrias, la cual en muchas especies se incrementa en longitud hacia la parte distal. Trocánteres sin muescas.

Familia Agelenidae (en parte) 40.b Tarsos con numerosas tricobotrias, pero distribuidas irregularmente. Trocánteres con una muesca curva en el borde distal en el lado ventral (escotado) (Fig. 27).

(40.b) 41.a Fila posterior de ojos tan fuertemente recurva que puede ser considerada que forma dos filas. Pieza anterior del lorum redondeada hacia atrás y encaja dentro de una muesca en la pieza posterior (Fig. 28). Saco de huevos transportados en las hilanderas y los juveniles transportados en el dorso de la madre.

Familia Lycosidae 41.b Fila posterior de ojos no formando dos filas distintas y levemente recurvas (Fig. 29). Pieza anterior del lorum con una muesca dentro del cual acopla la pieza posterior, o una sutura transversal entre las dos piezas. Saco de huevos cogido bajo el cefalotórax y los juveniles no son transportados por la madre.

Familia Pisauridae

(39.b) 42.a Clípeo de menor longitud que el área media ocular, en la mayoría de los casos. Forma de los ojos homogéneos.

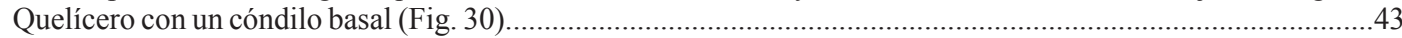
42.b Clípeo usualmente tan alto como la altura del área media ocular (Fig. 31). Forma de los ojos heterogéneos. Quelícero sin cóndilo basal.... 


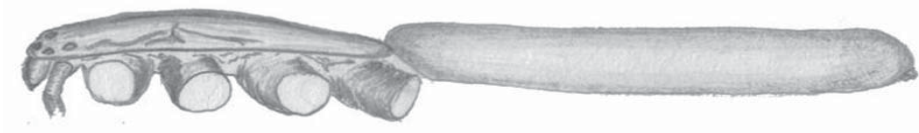

FIgURA 24. Doliomalus sp., cefalotórax y abdomen aplastado.

Figure 24. Doliomalus sp., flat carapace and abdomen.

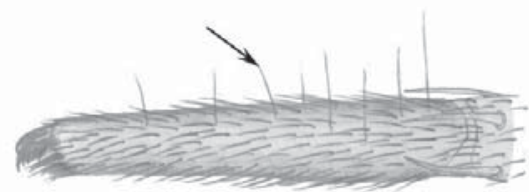

FigURA 25 Lycosa sp., tarso con tricobotrias.

FIGURE 25. Lycosa sp., tarsus with trichobotrias.

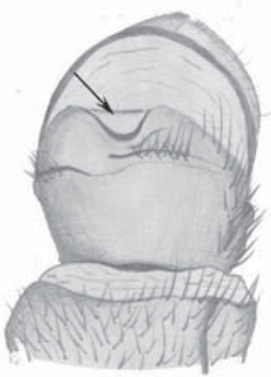

FiguRa 27. Lycosa sp., trocánter con una muesca curva.

FiguRE 27. Lycosa sp., trochanter with a curved notched.

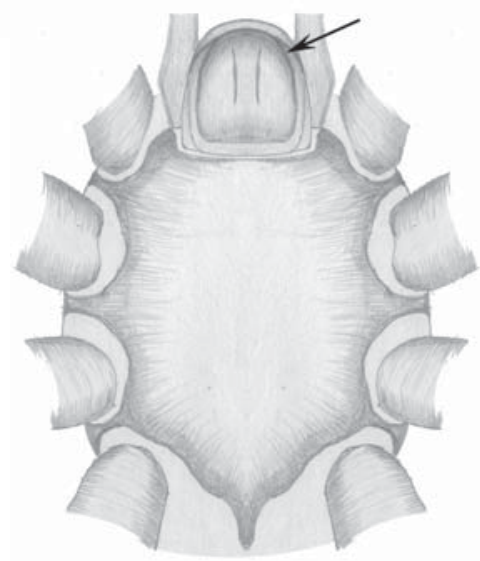

FIgURA 26. Tetragnathidae, labio rebordeado.

FIGURE 26. Tetragnathidae, rebordered labium.

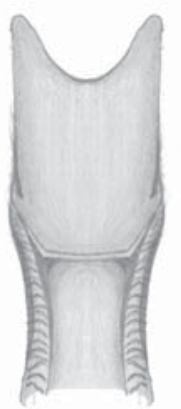

Figura 28. Lycosa sp., lorum del pedicelo.

Figure 28. Lycosa sp., lorum of pedicel.

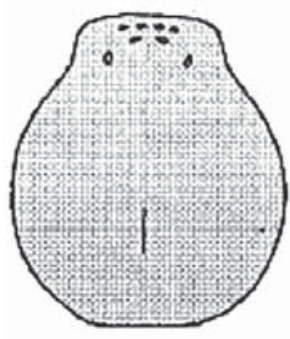

FIgURA 29. Pisauridae, disposición de ojos (Ex.: Ramírez 1999).

Figure 29. Pisauridae, eyes arrangement. (Ex.: Ramírez 1999).

(42.a) 43.a Surco epigástrico procurvo (Fig. 32). Cóndilo basal del quelícero rudimentario. Los quelíceros, en la mayoría de las especies, largos y poderosos (Fig. 33). Fémur con tricobotrias. Enditos palpales paralelos o ligeramente divergentes, en algunas especies ancho y truncado distalmente. Margen del quelícero con fuertes dientes. Ojos laterales separados de los ojos medianos por una distancia levemente superior al diámetro de éstos (excepto en Nephila sp., género no descrito para Chile).

Familia Tetragnathidae 43.b Surco epigástrico relativamente recto. Cóndilo basal del quelícero notorio y presente en la mayoría de las especies (Fig. 30). Todos los fémures sin tricobotrias, cuando presentes se encuentran en el fémur IV. Enditos ligeramente convergentes o paralelos, cortos, cuadrados y oblicuamente truncados. Ojos laterales a menudo separados de los ojos medianos por 2 a 3 veces su diámetro (excepto en algunas especies de los géneros Mecynogea y Cyclosa).

Familia Araneidae 
(42.b) 44.a Tibia IV en la mayoría de las especies con dos espinas dorsales; cuando ausentes, existe una espina corta en los metatarsos I y II. Tibia del palpo del macho sin apófisis (aun que puede estar dilatada distalmente). Tarso palpal de la hembra con una uña en la mayoría de las especies. Presencia de crestas estridulatorias en los quelíceros

Familia Linyphiidae 44.b Sin la combinación de caracteres anteriores. Cono labral presente detrás del quelícero. Escuto presente y fuertemente esclerosado o al menos con puntos dorsales coriáceos. Pedicelo surge desde una cavidad contorneada circular en el declive posterior del cefalotórax. Longitud del cuerpo 1 a 1,3 $\mathrm{mm}$.

Familia Anapidae

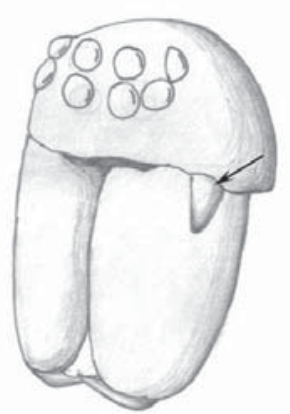

Figura 30. Araneidae, clípeo y cóndilo basal del quelícero.

Figure 30. Araneidae, clypeus and boss on chelicerae.

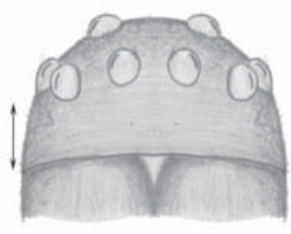

Figura 31. Linyphiidae, clípeo y ojos.

Figure 31. Linyphiidae, clypeus and eyes.

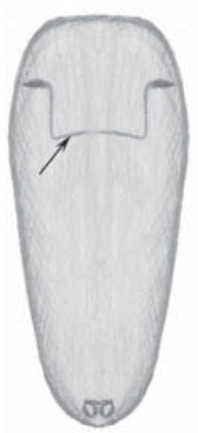

Figura 32. Tetragnathidae, surco epigástrico procurvo.

Figure 32. Tetragnathidae, epigastric furrow procurved.

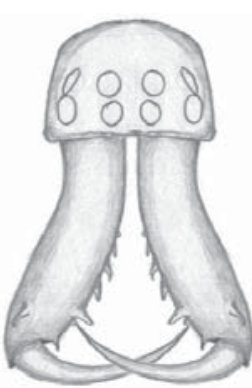

FigURA 33. Tetragnathidae, quelíceros y espinas.

Figure 33. Tetragnathidae, chelicerae and spines.

TABLA I: Familias de arañas presentes en Chile.

TABLE I: Spiders families present in Chile

\begin{tabular}{llll}
\hline \multicolumn{2}{c}{ Infraorden Araneomorphae } & & \multicolumn{1}{c}{ Infraorden } \\
& & & Mygalomorphae \\
\hline Agelenidae & Hahnidae & Pisauridae & Actinopodidae \\
Amaurobiidae & Linyphiidae & Salticidae & Dipluridae \\
Amphinectidae & Lycosidae & Scytodidae & Hexatelidae \\
Anapidae & Malkaridae & Segestriidae & Migidae \\
Anyphaenidae & Mecysmaucheniidae & Sicariidae & Nemesiidae \\
Araneidae & Micropholcommatidae & Sparassidae & Theraphosidae \\
Austrochilidae & Mimetidae & Synotaxidae & \\
Caponiidae & Miturgidae & Tengellidae $*$ & \\
Clubionidae $*$ & Nesticidae & Tetragnathidae & \\
Corinnidae & Oecobiidae & Theridiidae & \\
Ctenidae $*$ & Oonopidae & Thomisidae & \\
Desidae & Orsolobidae & Titanoecidae & \\
Dictynidae & Oxyopidae $*$ & Trochanteriidae & \\
Diguetidae & Palpimanidae & Uloboridae & \\
Dysderidae & Philodromidae & Zodariidae & \\
Filistatidae & Pholcidae & Zoridae & \\
Gnaphosidae & & & \\
\hline
\end{tabular}

* Familias posiblemente presentes en Chile. 


\section{ANTECEDENTES BIOLOGICOS DEFAMILIAS PRESENTESENCHILE}

Agelenidae (TEJedoras de EMBudo)

La mayoría de estas arañas tejen una tela en forma de lámina horizontal o de plataforma que se va cerrando por la parte superior en una especie de tubo o embudo, el cual se prolonga hasta uno de los extremos de la tela. Comúnmente pueden ser encontradas cerca del suelo, asociadas a matorrales bajos, hierbas, entre otros. Su tamaño puede variar desde unos pocos milímetros hasta aproximadamente $10 \mathrm{~mm}$.

Para Chile se ha citado Tegenaria pagana, con una amplia distribución en América, Europa, Asia central, Nueva Zelanda y Chile; Tegenaria domestica, con distribución cosmopolita, posiblemente presente en Chile (Platnick 2005).

AmaurobiIdae

Desde esta familia se han transferido varios géneros a familias como Amphinectidae, Desidae y Titanoecidae (Lehtinen 1967; Leech 1972 fide Roth 1993). Ahora autores como Roth (1993) incluyen especies de Desidae y Titanoecidae dentro de los Amaurobiidae cuando elabora su clave dicotómica. Esto nos da indicios que existen especies de los Amphinectidae, Desidae y Titanoecidae que pueden sobreponerse con los caracteres utilizados para separar los Amaurobiidae. Por otro lado se menciona que los Amaurobiidae semejan en apariencia a arañas ecribeladas como los Agelenidae (Kaston 1978).

Estas arañas construyen telas bastante complejas con muchos hilos entrecruzados y con un refugio circular. Para Chile se han citado ocho géneros: Anisacate, Callevopsis, Emmenomma, Hicanodon, Livius, Macrobunus, Neoporteria y Rubrius. Neoporteria sólo se ha descrito para el territorio nacional (Platnick 2005, Ramírez et al. 2004).

\section{AMPHINECTIDAE}

Se han reconocido dos género en Chile: Metaltella y Calacadia, este último con siete especies descritas sólo para Chile (Platnick 2005).

\section{ANAPIDAE}

Las arañas de esta familia construyen telas orbiculares de alrededor de $7 \mathrm{~cm}$ de diámetro por 10 de alto. En algunos casos, existe un eje central que permite que la tela sea más o menos cóncava y en otros casos existe una supresión de las últimas espirales (Watson \& Dallwitz 2004).

Se han descrito seis géneros para Chile: Crassanapis, Elanapis, Minanapis, Pecanapis, Sheranapis y Sofanapis. Sólo los últimos tres han sido descritos exclusivamente para el país (Platnick \& Forster 1989, Ramírez et al. 2004).

\section{ANYPHAENIDAE (ARAÑAS FANTASMAS)}

Las especies pertenecientes a esta familia no fabrican telas como trampas para capturar su alimento, sino que acechan, persiguen y capturan sus presas preferentemente sobre el follaje de árboles y arbustos; raramente son encontradas en el suelo. Su coloración puede ser muy variada, pero es común encontrar especímenes de colores amarillo o pardo pálido con franjas pardas o grises sobre el abdomen.

Para Chile se han descrito 22 géneros y alrededor de 65 especies: Acanthoceto, Amaurobioides, Anyphaenoides, Araiya, Axyracrus, Aysenia, Aysenoides, Coptoprepes, Ferrieria, Gamakia, Gayenna, Gayennoides, Malenella, Monapia, Negayan, Oxysoma, Phidyle, Philisca, Sanogasta, Selknamia, Tasata y Tomopisthes. El género Philisca está representado por 13 especies en Chile, de las cuales $P$. ingens y $P$. ornata son propias de Juan Fernández (Ramírez 1995, 1997, 2003). La especie Wulfila scopulatus con distribución en América, posiblemente presente en Chile (Platnick 2005).

Araneidae (tejedoras orbiculares o ARañas de JARDÍN)

Los araneidos constituyen una de las familias más frecuentemente recolectadas, debido a que se presentan comúnmente en el interior de habitaciones humanas y jardines. Son fácilmente reconocibles por hacer una tela orbicular relativamente perfecta, con sus radios y espiras bien definidas y, en algunos casos, con pequeños adornos blanquecinos en el centro de la tela.

En Chile se han reconocido 12 géneros: Araneus, Cyclosa, Gasteracantha, Heterognatha, Mastophora, Mecynogea, Metepeira, Molinaranea, Nicolepeira, Ocrepeira, Parawixia y Ursa (Platnick 2005). Las especies Argiope trifasciata y Neoscona nautica con distribución cosmopolita y cosmotro-pical respectivamente, es posible que estén presentes en Chile. El género Zygiella no 
está mencionado en el catálogo para Chile; sin embargo en recolectas del primer autor de este trabajo se ha determinado la presencia de ejemplares de este género en nuestro territorio y se supone que ha sido introducida.

\section{Austrochilidae}

Los representantes de esta familia construyen telas horizontales muy notorias, relativamente irregulares y comúnmente encontradas en los bosques del sur de Chile y Argentina. Durante el día las arañas se refugian en una parte de la tela que tiene forma de cono. Se recomienda la recolección de estas arañas en la noche, cuando caminan por debajo de su tela. Familia con dos géneros en Chile: Austrochilus y Thaida. (Forster et al. 1987, Grismado \& Lopardo 2003 a, Lopardo et al. 2004, Zapfe 1955).

CAPONIIDAE (ARAÑAS DE DOS OJOS)

Es la única familia presente en Chile cuyos individuos poseen sólo dos ojos.

Para el país se han citado: Caponina chilensis, Notnops calderoni, Taintnops goloboffi, Tisentnops leopoldi (Platnick 2005).

Clubionidae (arañas de Saco)

En Chile, según el catálogo mundial de arañas (Platnick 2005), estaría presente el género Clubiona con 9 especies. Estas especies fueron descritas por Nicolet (1849); sin embargo, los holotipos están extraviados, por lo que no se ha podido revisar y corroborar su posición taxonómica (Ramírez com. pers. 2005).

Corinnidae

Grupo bastante complejo, porque muchos de sus géneros y especies han sido transferidos recientemente de familia. Son arañas cazadoras activas. Pueden ser halladas asociadas al follaje o al suelo.

La fauna de corinnidos en Chile está representada por cuatro géneros: Meriola, Olbus, Trachelas y Trachelopachys. Olbus sólo se cita para nuestro país (Ramírez et al. 2001). Las especies Abapeba kochi en Sudamérica y Creugas gulosus con distribución cosmopolita, es posible que estén presentes en el país (Platnick 2005).

CTENidAe (ARAÑAS ERRANTES)

Estas arañas cazan sus presas en el follaje o en el suelo (Watson \& Dallwitz 2004). Son fácilmente distinguibles por una disposición 2-4-2 de los ojos. La distribución de esta familia aun es dudosa; Platnick (2004) señala que Ctenus walckenaeri probablemente estaría presente en Sudamérica y Phoneutria boliviensis en América Central y Sudamérica; sin embargo, hasta la fecha no se han obtenido ejemplares proveniente del territorio nacional.

Desidae

Lehtinen (1967) y Leech (1972 fide Roth 1993) consideran a los Desidae como una subfamilia de los Amaurobiidae. En tanto, Roth (1993) incluye varias especies de esta familia en Amaurobiidae y otras las deja en la familia Desidae para efectos de elaborar su clave de géneros de Norteamérica. Actualmente este es un taxon válido (Platnick 2005). Para Chile se ha descrito sólo una especie, Porteria albopunctata (Platnick op. cit.).

Dictynidae (TEJedoras de mallas)

Arañas constructoras de redes horizontales, muy difusas y generalmente entre las inflorescencias de las plantas o entre las hojas. La mayoría son de pequeño tamaño, no superando los $4 \mathrm{~mm}$ (Watson \& Dallwitz 2004).

En Chile se han reconocido cuatro especies: Dictyna togata, Dictyna trivirgata, Tahuantina zapfeae, Thallumetus acanthochirus (Lehtinen 1967, MelloLeitão 1943).

Diguetidae

Para Chile se han descrito sólo dos especies: Segestrioides copiapo y S. tofo (Platnick 2005).

DYSDERIDAE

Arañas cazadoras nocturnas, persiguen activamente sus presas, por lo que no construyen redes para atrapar su alimento. Las telas son utilizadas para construir un refugio en forma de tubo, generalmente bajo rocas. Los huevos son puestos en un cocon bastante transparente, y una vez eclosionados los juveniles viven por un tiempo con la madre. Frecuentemente se encuentran bajo las cortezas de los árboles, entre el musgo, bajo rocas y en lugares húmedos y oscuros.

En Chile se ha encontrado la especie Dysdera crocata (cosmopolita) (Forster \& Platnick 1985). Esta especie es fácilmente reconocible por la coloración rojo-anaranjada del cefalotórax y cremablanquecino en el abdomen y por presentar 
quelíceros largos y considerablemente proyectados hacia delante.

Filistatidae (TEJedoras En GRIETAs)

Construyen un refugio tubular, en el cual se esconden. Generalmente construyen su tela en pequeñas fisuras de las paredes o grietas del terreno. Pueden presentar un cefalotórax de color amarillo claro y reticulado; abdomen amarillo claro, opaco y con bandas transversales pardas; patas con anillos negros incompletos. Una especie, Filistatoides milloti, recolectada en Tal Tal y la Quebrada de la Plata (Zapfe 1961f).

GNAPHOSIDAE (ARAÑAS SIGILOSAS DEL SUELO O ARAÑAS DE SUELO)

Cazadoras activas, persiguen a sus presas cautelosamente por el suelo. Se alimentan principalmente de otras arañas. Reconocibles por tener hilanderas cilíndricas, siendo las anteriores muy esclerosadas y separadas por una distancia similar al diámetro de una de ellas, lo cual deja ver libremente las hilanderas medias. Pueden ser confundidas con algunos Agelenidae, pero éstos tienen tres uñas tarsales y los Gnaphosidae sólo dos.

Existen seis géneros presentes en el país: Apodrassodes, Apopyllus, Camillina, Drassodes, Echemoides y Eilica (Platnick 1983, Platnick \& Shadab 1983a, 1983b). Las especies Scotophaeus blackwalli y Urozelotes rusticus con distribución cosmopolita, posiblemente presentes en el país.

\section{HAHNIIDAE}

Son constructoras de telas laminares, delicadas y pequeñas, sin un refugio y comúnmente asociadas al follaje (Watson \& Dallwitz 2004, Ramírez com. pers. 2005). Son fácilmente reconocibles por la disposición de las hilanderas en una fila que tiende a ser transversal.

Dos géneros y cuatro especies citadas para nuestro país: Cybaeolus delfini, C. pusillus, C. rastellus, Hahnia michaelseni (Lehtinen 1967).

LINYPHIIDAE (ARAÑAS ENANAS, TEJEDORAS DE LÁMINAS O TEJEDORAS DE LÍNEAS)

La tela tiene forma de una lámina horizontal o de domo y en la mayoría de los Linyphiidae es de gran extensión. No construyen estructuras como refugio, para mudar o poner los huevos. Se dispersan rápidamente y en general se pueden encontrar errando o cazando, más que en sus propias telas. Poseen patas largas y delgadas provistas de finas espinas. Se diferencian de los Araneidae por la ausencia del cóndilo basal queliceral y porque en algunas especies presentan en la superficie lateral del quelícero una fila horizontal de estrías que correspondería al área estridulante.

Es una de las familias con mayor número de géneros citados para Chile con: 31 géneros y alrededor de 95 especies. Varias especies son endémicas de Juan Fernández. Los géneros que presentan mayor número de especies en Chile son: Neomaso, Laminacauda y Dubiaranea (Platnick 2005).

LYCOSIDAE (ARAÑAS LOBO)

Arañas errantes, corredoras en el suelo; son cazadoras activas, no construyen redes para cazar y utilizan la tela para revestir sus refugios (comúnmente orificios en el suelo) y para construir la ooteca, que es transportada por la hembra en sus hilanderas. También es frecuente encontrarlas bajo pequeñas rocas, especialmente donde exista humedad alta o cercanas al agua. Muchas veces se pueden observar sobre el espejo de agua y desplazándose sobre la superficie, ya que poseen tarsos con abundantes pelos y cerdas hidrófugas. Una vez que eclosionan los huevos, los juveniles son llevados en el dorso de la madre durante un tiempo considerable. La coloración de las distintas especies varía de pardo a grisáceo con franjas negras o pardo oscuras, patrones de coloridos abdominales dorsales bastante estables entre las especies chilenas, lo que permite una fácil identificación.

Se reconocen tres géneros y diez especies en Chile: Allocosa, Lycosa y Pardosa (Casanueva 1980).

\section{Malkaridae (ARAÑAS ESCUdo)}

Esta familia fue elevada desde su previo estatus de subfamilia de Araneidae por Wunderlich (1986 fide Platnick \& Forster 1987).

La única especie descubierta en nuestro país es Chilenoides australis (Grismado \& Lopardo 2003 b) y se puede reconocer por la particular forma del cefalotórax, el cual presenta grandes tubérculos sobresaliente en su margen, desde la coxa II hasta la IV (Fig. 34), este ultimo carácter también lo hace diferenciarse de Sternodes y Carathea, géneros pertenecientes a esta familia (Platnick \& Forster op. cit.). 


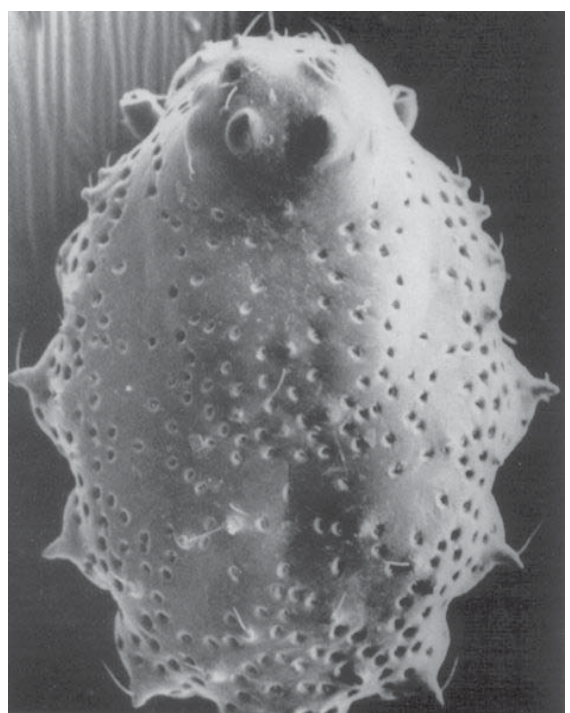

Figura 34. Chilenoides australis, cefalotórax de la hembra en vista dorsal mostrando las alveolaciones y tubérculos (Ex.: Platnick \& Forster 1987).

FIGURE 34. Chilenoides australis, female carapace, dorsal view, showing alveolations and tubercles (Ex.: Platnick \& Forster 1987).

\section{Mecysmauchenitidae}

Los miembros de esta familia se reconocen fácilmente por tener sólo un par de hilanderas desarrolladas, y las restantes reducidas sólo a la fúsula; porque el quelícero nace desde un foramen; por la particular forma del cefalotórax, casi rectangular; y la parte cefálica muy alta, proyectada hacia arriba en vista lateral.

En Chile se han encontrado cinco géneros. Mecysmauchenius con 16 especies en el país y los restantes monoespecíficos Chilarchaea quellon, Mecysmauchenioides nordenskjoldi, Mesarchaea bellavista y Semysmauchenius antillanca (Forster \& Platnick 1984, Grismado \& Lopardo 2003 b).

\section{MicROPHOLCOMMATIDAE}

Esta familia es incluida dentro de la clave dicotómica pese a que no se pudo obtener ejemplares de comparación; no obstante se incluyen las fotografías originales de la descripción de especies presentes en Chile, donde se muestra los caracteres que permiten reconocer esta familia (Figs. 36-39).

Se han reconocido sólo dos especies, Teutoniella cekalovici y Tricellina gertschi en nuestro país (Platnick \& Forster 1986).

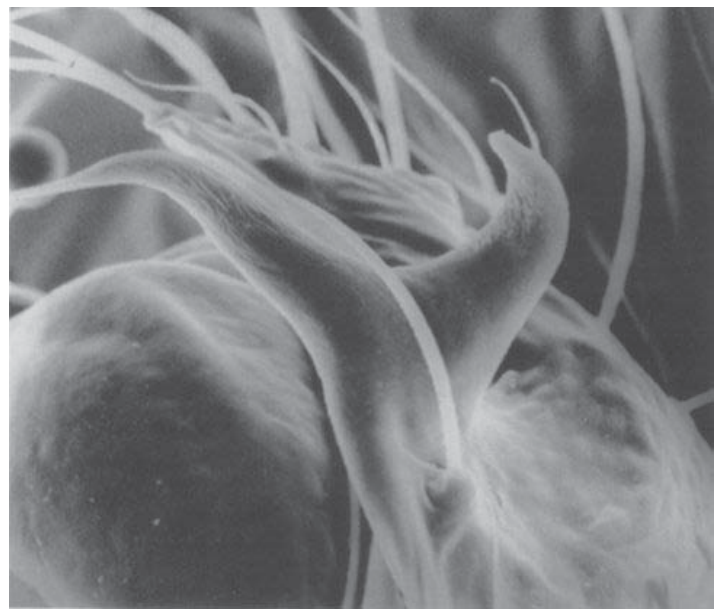

Figura 35. Chilenoides australis, macho; paracimbo del palpo derecho, vista retrolateral (Ex.: Platnick \& Forster 1987)

FIGURE 35. Chilenoides australis, male; paracymbium of the right palp, retrolateral view (Ex.: Platnick \& Forster 1987).

Mimetidae (ARAÑAS PIRATA)

Estas arañas no son constructoras de redes para atrapar sus presas; frecuentemente se menciona que son predadoras de otras arañas, pero se les ha reportado comiendo insectos; pueden ser encontradas sobre la tela de otras arañas (generalmente en arbustos) en donde imitan las vibraciones producidas por las presas en los hilos y cuando la araña dueña de la tela va a capturar la supuesta presa el mimetido la atrapa y devora (Watson \& Dallwitz 2004).

Hay cuatro géneros en nuestro país: Ero, Gelanor, Gnolus y Oarces. La mayoría de las especies presentes en nuestro territorio tienen el abdomen relativamente triangular con la parte anterior más ancha, en Oarces reticulatus esto no es tan marcado, pero la prolongación posterior y su combinación de colores (blanco, pardo, negro) los distingue (Platnick \& Shabad 1993)

\section{MitURGIDAE: (ARAÑAS MERODEADORAS)}

Esta familia es algo compleja por que muchos de sus géneros has sido transferidos desde otras familias y en su mayoría desde los Clubionidae. Se les suele confundir fácilmente con Clubionidae.

En Chile con un sólo género, Macerio, con ocho especies (Ramírez et al. 1997). 

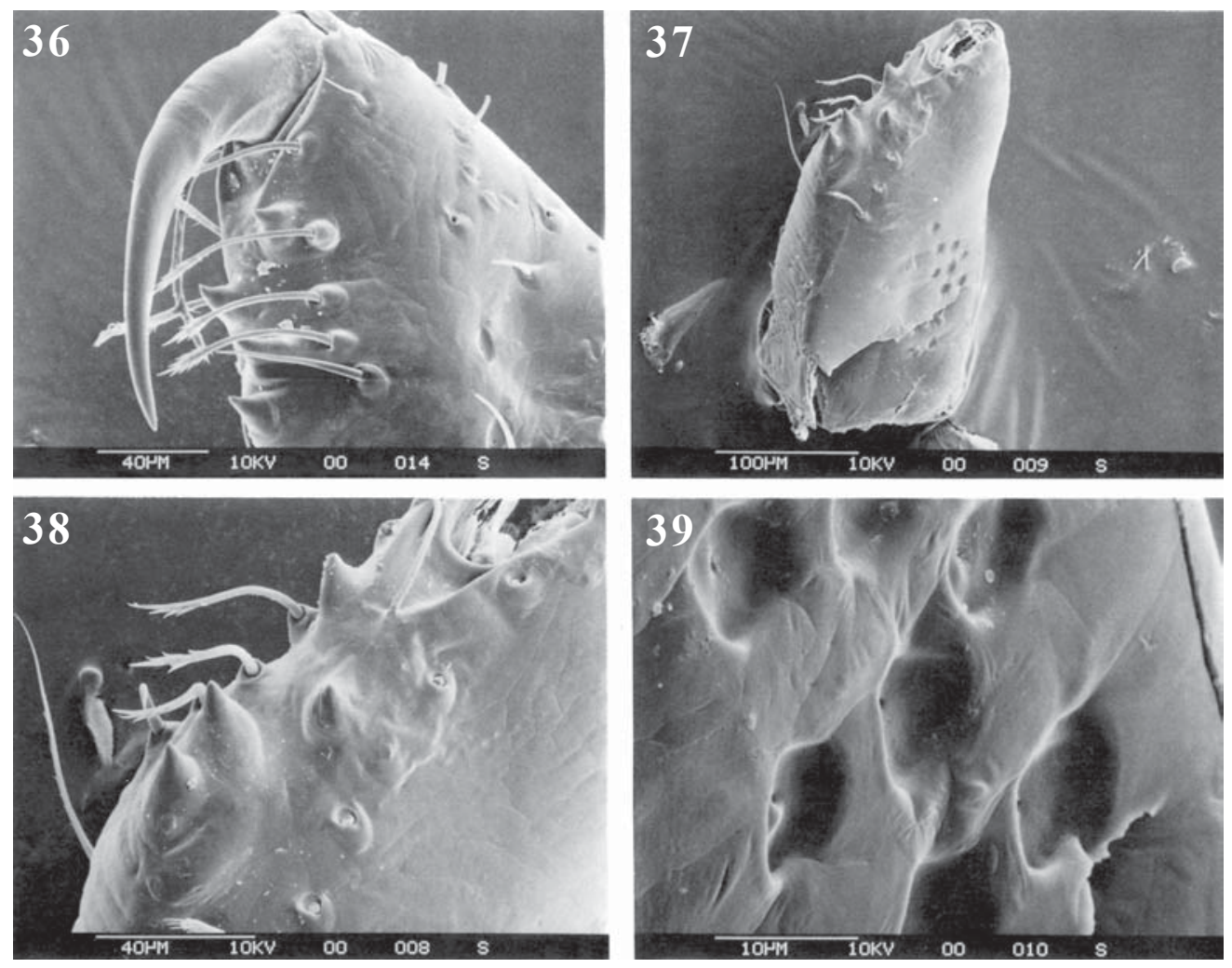

FIGURAS 36-39. Teutoniella cekalovici, macho. 36. Quelícero derecho vista anterior; noten la seta promarginal proximal, recta y rígida. 37. Quelícero izquierdo, vista oblicua posteroventral; noten las depresiones proximales circulares. 38. El mismo; noten la glándula queliceral elevada en un montículo fusionada al diente promarginal más proximal. 39. El mismo; depresiones circulares proximales, mostrando los poros (Ex.: Platnick \& Forster 1986).

FIGURES 36-39: Teutoniella cekalovici, macho. 36. Right chelicera, anterior view; note straight, staff, proximal promarginal seta. 37. Left chelicera, oblique posteroventral view, note circular proximal depressions. 38. Same; note elevated cheliceral gland mound fused to most proximal promarginal tooth. 38. Same, circular proximal depressions, showing pores (Ex.: Platnick \& Forster 1986).

NESTiCidAe (ARAÑAS TEJEDORAS DE CUEVAS O SÓTANOS) Generalmente tejen redes irregulares en grietas, entre las rocas o lugares oscuros como cuevas, entre otros. La ooteca es transportada en las hilanderas y soportada por la pata IV (Watson \& Dallwitz 2004). Se pueden diferenciar de los Theridiidae, porque los enditos palpales son casi paralelos (en Theridiidae fuertemente convergentes). Palpo del macho con un paracimbio grande y notorio. Hembra uña del palpo muy grande .

Sólo una especie descrita para el país, Nesticus delfini (Platnick 2005).

Oecobildae (aRAÑas tejedora de Redes planas) Estas cribelatadas pueden ser encontradas bajo rocas. Característico es el tubérculo anal con una fila de setas que lo rodea y la forma general del cuerpo.

Las especies Oecobius cellariorum y O. navus, ambas con distribución cosmopolita, probablemente presentes en Chile (Platnick 2005).

Oonopidae (ARAÑas ENANAS DE SEIS OJos)

Cazadoras nocturnas, no construyen tela. Se mencionan como carroñeras de nidos de aves y telas de otras arañas. De pequeño tamaño, no superando los $3 \mathrm{~mm}$ (Watson \& Dallwitz 2004).

Cuatro especies en el país: Unicorn catleyi, U. chacabuco, U. socos y U. toconao (Platnick \& Brescovit 1995). 
ORSOLOBIDAE

Arañas de tamaño medio a pequeño, las más grandes $7 \mathrm{~mm}$. Cazadoras errantes, encontradas generalmente en la hojarasca o los musgos. La ooteca es construida dentro del refugio y es plana a convexo, generalmente colocada lejos de la entrada. Los juveniles son activos durante el invierno, pero rara vez mudan durante esos meses. Cuatro géneros presentes en el país: Chileolobus, Mallecolobus, Orsolobus y Osor-nolobus; con un total de treinta especies (Forster \& Platnick 1985).

OXYOPIDAE (ARAÑAS LINCE)

No construyen redes para capturar sus presas, como refugio, para mudar o como criadero. Corren muy rápido y hasta pueden saltar. Se distinguen fácilmente por la distribución de los ojos.

Esta familia se incluye dentro de la clave porque Mello- Leitão (1943) descubrió una especie para el país, Peucetia roceonigra; sin embargo, Santos \& Brescovit (2003 fide Platnick 2005) actualmente la coloca como sinonimia de $P$. rubrolineata que se distribuye desde Panamá hasta Argentina. Platnick (2005) no deja claro si está presente en Chile. Además no se pudo revisar el ejemplar determinado por Mello-Leitão (op. cit.) y comprobar su procedencia.

PALPIMANidAe

El cefalotórax de las arañas perteneciente a esta familia es fuertemente esclerotizado, con muchas rugosidades y tubérculos. Usualmente la fovea es muy notoria.

Los géneros presentes en Chile son: Anisaedus (con una especies $A$. pellucidas) y Notiothops con ocho especies (Platnick et al. 1999, Zapfe 1961); todas las especies con ocho ojos.

Philodromidae (ARAÑAs CANGReJo CORREdoras)

Los adultos no construyen telas, son cazadoras activas, persiguiendo a su presa entre el follaje. Son capaces de moverse muy rápidamente.

En Chile, Tibellus chilensis y Petrichus con cuatro especies (Mello-Leitão 1943, Platnick 2005)

Pholcidae (ARAÑAs de los SÓtanos o Arañas típUla) Arañas fácilmente reconocibles por la longitud de sus patas, siendo cuatro a cinco veces más largas que la longitud del cuerpo; con los metatarsos aparentemente segmentados, por que son capaces de curvarlo en gran medida; arañas de pequeño tamaño, longitud total 1-15 mm; usualmente con 8 ó 6 ojos, el patrón de los ojos puede ser muy variable, pero lo más común es ojos dispuestos en dos tríos, en donde se juntan los anteriores laterales con posteriores laterales y posteriores medios, quedando libres solamente los anteriores medios; algunas especies pueden tener sólo seis ojos, los anteriores medios ausentes. Construyen una tela irregular muy desordenada, en la cual se ubican por debajo en posición invertida. Transportan la ooteca en los quelíceros y ésta es hecha con hilos entrecruzados y enlazados muy sueltamente. Pueden ser encontradas en sótanos o lugares oscuros (Huber 2000).

Para Chile se han descrito cuatro géneros, con un total de 10 especies: Aucana. Chibchea, Mesabolivar, Nerudia. Las especies Crossopriza lyoni, Pholcus phalangioides, Physocyclus globosus y Smeringopus pallidus con distribución cosmopolita; Litoporus uncatus distribuido en Sudamérica; Modisimus culicinus en Norteamérica, Sudamérica, El Congo, Hawai, Islas Marshall y Seychelles; Pholcus ancoralis distribuido en las islas del Pacífico sur y Hawaii, todas especies posiblemente presentes en el país (Platnick 2005, Huber 2000).

Pisauridae (ARAÑAS CONSTRUCTORAS DE GUARDERÍA O ARAÑAS BALSA)

En apariencia son similares a las arañas lobo (Lycosidae), pero se diferencian porque la segunda fila de ojos no es fuertemente recurva. Son cazadoras activas, persiguiendo y corriendo tras sus presas sobre el suelo, a veces pueden ser encontradas asociadas a agua. El género Dolomedes puede andar sobre el agua y en algunos casos capturar peces pequeños. Las hembras construyen una tela con estructura especial (guardería) para la protección de los juveniles; además transporta la ooteca bajo el cefalotórax tomada con los quelíceros y los pedipalpos.

Sólo una especie descrita para el país, Dolomedes pullatus (Platnick 2005).

SALTiCidAe (ARAÑAS SALTARINAS O CAZA MOSCAS)

Estas arañas son bastante conocidas por su aguda visión, la que le permite ir tras su presa y capturarla. No son constructoras de telas. Durante el día se las puede encontrar cazando en el follaje, en el suelo, en las paredes, entre otros. Construyen refugios para mudar, hibernar y para pasar la noche entre las cortezas, entre rocas o enrollando hojas. 
En Chile se han reconocido siete géneros con 15 especies en total: Admesturius, Atomosphyrus, Dendryphantes, Euophrys, Hurius, Trydarssus y Tullgrenella (Galiano 1980, 1985, 1988). Las especies Hasarius adansoni y Plexippus paykulli tienen distribución cosmopolita y posiblemente podrían estar en Chile.

SCYTODIDAE: (ARAÑAS ESCUPIDORAS, ARAÑAS DE PATAS LARGAS, ARAÑA TIGRE O ATIGRADA DE LOS RINCONES) Estas arañas tienen un enorme desarrollo de las glándulas de veneno que ocupa una gran parte del cefalotórax. La glándula esta dividida en dos, una pequeña parte en el frente que es la que produce el veneno y una porción más grande posterior que secreta una sustancia mucilaginosa (Kaston 1978). Para capturar su alimento escupe a su presa con el líquido pegajoso que secretan por sus quelíceros, inmo-vilizándola. Son depredadoras de otras arañas. Comúnmente pueden ser encontradas asociadas a las viviendas humanas, pero también en el exterior, como por ejemplo, en bosques.

Se ha descrito una especie para Chile: Scytodes globula (Nicolet 1849).

\section{Segestriidae (arañas tubo)}

Arañas muy particulares, ya que construyen una tela en forma cilíndrica en un orificio en la corteza de los árboles, en las paredes, sobre las rocas o cualquiera hendidura. La parte exterior de la tela queda con varios hilos adosados al sustrato (como un cono) que permiten detectar los posibles insectos presa; las arañas esperan dentro del cono y capturan sus presas con los tres primeros pares de patas. Estas arañas tienen las coxas III proyectadas hacia delante, lo que se atribuye a su modo de vida y forma de captura de las presas.

Dos especie en Chile: Ariadna maxima (típica araña tubo) y Segestria pusilla (Nicolet 1849).

SicARIIDAe (ARAÑAS SICARIO, ARAÑA DE LOS RINCONES) Esta familia posee dos géneros, Sicarius y Loxosceles. Las primeras son conocidas como arañas sicario, ya que se camuflan, acechan y capturan a sus presas. Generalmente se encuentran bajo rocas, en lugares áridos a semiáridos o lugares donde puedan camuflarse con la tierra. Se menciona que muchas veces ellas mismas se entierran o tiran arena sobre su cuerpo para camuflarse, aun cuando sus colores pasan desapercibidos en el medio.
Para Chile se citan ocho especies del género Sicarius (Gerschman \& Schiapelli 1979).

Loxosceles, comúnmente conocidas como araña de los rincones, han recibido mucha publicidad debido a numerosos casos de envenenamiento ocurridos en el país. Estas arañas se encuentran frecuentemente asociadas a las habitaciones humanas, en lugares secos, relativamente tranquilos, oscuros y sin mayor ruido (e.g., detrás de los cuadros, en los armarios, detrás de libro, entre otros). No hacen telas para cazar sus presas, sólo fabrican una tela irregular, muy sedosa y algodonosa como refugio, en donde depositan su ooteca, la cual es un disco plano a cóncavo más o menos translúcido. Son cazadoras nocturnas activas. Las especies presentes en el país corresponderían a $L$. laeta, $L$. coquimbo y L. rufescens, esta última designada como cosmopolita (Gertsch \& Ennik 1983, Canals et al. 2004).

Sparassidae (Arañas cangrejo gigantes o arañas VERDES)

El nombre Sparassidae tiene prioridad sobre Heteropodidae, pero algunos autores prefieren llamar a esta familia Heteropodidae (Kaston 1978, Roth 1993). Los adultos no construyen tela para capturar sus presas, sino que aguardan colgando de la vegetación baja en lugares cubiertos de hierba y cuando pasa un insecto saltan sobre él dejándose caer al vacío.

Cuatro especies en Chile: Olios flavens, O. ventrosus, Polybetes delfini y P. martius (Nicolet 1849, Platnick 2005).

\section{Synotaxidae}

Pueden ser separados de otras familias por una incisión en el margen cimbial retrolateral y un paracimbo excavado en el palpo del macho. Esclerito palpal situado terminalmente, fémur basalmente engrosado, espuela en la tibia y patela, abdomen del macho modificado anteriormente. Estas características suelen ser utilizadas, pero pueden ser compartidas con otros taxa relacionados u ocurren esporádicamente en la familia. Para una descripción de la alta variabilidad de esta familia ver Griswold $e t$ al. (1998).

Se han descrito dos géneros para Chile: Physoglenes con cuatro especies y Chileotaxus con una $C$. sans. Para diagnosis y descripción de estas especies ver Forster et al. (1990). 
Los Synotaxidae no han sido incluidos en la clave propuesta porque se tiene sólo los caracteres diagnósticos para los machos y por ser una familia muy variable.

Tengellidae

Se reconocen por ser arañas con ocho ojos; cribelo y calamistro presentes; Trocánteres III y IV con una muesca moderada; tarso escopulado (Roth 1993).

Esta familia es validada por Wolff (1977). Anteriormente Lehtinen (1967) la considera una subfamilia de los Miturgidae y la caracteriza por presentar dos uñas tarsales y el labio con una muesca distal. Sin embargo, todas las especies de Tengella tienen tres uñas tarsales, el labio con una muesca en la base y un patrón ventral de espinas en la tibia y el metatarso distintos de la caracterización de Lehtinen. Por otro lado se menciona que Zorocrates (Lycosoidea: Zoropsidae) sería un género relacionado con Tengella (Amaurobioidea: Tengellidae) (Wolf op . cit.). Pero estudios filogenéticos recientes demuestran que esta familia sería polifilética (Griswold 1993, Griswold et al. 1999).

El holotipo del género Tengella es T. perfuga descrito por Dahl (1901, fide Platnick 2005) basado en una hembra proveniente de Sudamérica, sin indicar con precisión la localidad geográfica donde se recolectó. Este espécimen fue depositado en el Museo de Berlín, pero se ha reportado como perdido (Wolf op. cit.).

Al desconocer el tipo y no tener la seguridad que está presente en Chile, esta familia no se ha incluido en la clave propuesta en este estudio.

Tetragnathidae (TeJedoras orbiculares De QUELÍCEROS GRANDES)

Arañas fácilmente reconocibles por fabricar telas orbiculares muy notorias, generalmente en lugares húmedos o cercanos a cursos de aguas, entre la vegetación de pantanos o humedales (entre los juncos), con los hilos horizontales o en ángulo. Otra característica muy notoria a simple vista en la mayoría de las especies son sus poderosos quelíceros proyectados hacia delante, con diversas orna-mentaciones como espinas o setas. Cinco géneros presentes en el país: Chrysometa, Diphya, Dolichognatha, Metabus y Tetragnatha (Nicolet 1849, Archer 1963).
Nephilengys cruentata en Africa tropical y América, posiblemente presentes en Chile.

Therididdae ("CoBweb WeAvers")

Construyen tela para capturar a sus presas. Tela irregular y compleja, usualmente tiran los hilos laterales de seda y los reúnen en el centro entrecruzándolos. Las arañas se suspendes sobre su tela en posición invertida, mientras aguardan por su presa. Son de abdomen globoso y de patas delgadas, con una apariencia muy esbelta y elegante.

En Chile existen doce géneros: Achaearanea, Anelosimus, Chrysso, Dipoena, Episinus, Helvibis, Latrodectus, Paratheridula, Phoroncidia, Selkirkiella, Steatoda y Theridion (Levi 1967, Roth 1993, Agnarsson 2004). Desde un punto de vista médico se destaca el género Latrodectus. Estas arañas son comúnmente conocidas como "arañas del trigo, arañas de poto colorado o viudas negras”, entre muchos otros nombres. Presentan una coloración particular, siendo de cuerpo completamente negro y brillante, aterciopelado, con manchas rojas, naranjas, amarillo-anaranjado o carmesí en la parte dorsal y ventral del abdomen, estas manchas pueden ser circulares, ovales, en forma de flecha, luniformes u otras formas propias.

Coleosoma blandum, Theridula gonygaster y $T$. opulenta con distribución cosmopolita, posiblemente especies presentes en Chile.

ThOMISIDAe (ARAÑAS CANGREJO)

Los adultos no construyen redes para capturar su presa, sino que esperan que ésta llegue a ellos y capturan con el par de patas anteriores, atrapándolas con las espinas. No construyen telas como refugio, para mudar o hibernar. Pueden ser encontradas en el suelo, en la vegetación y frecuentemente sobre las flores. A menudo tienen colores muy crípticos.

En Chile existen seis géneros: Coenypha, Mecaphesa, Misumenoides, Philogaeus, Stephanopis y Tmarus (Mello-Leitão 1926, 1943). Distintivos los géneros Coenypha por la forma del cuerpo que semeja un trozo de madera, y Misumenoides por la coloración alrededor de los ojos en forma de antifaz. Actualmente algunas especies de Tomisus están incorporadas como Nomina dubia. Las especies Onocolus latiductus y Synema spirale distribuidas en Sudamérica, posiblemente presentes en Chile (Platnick 2005). 
TitANOECIDAE

Familia compleja de determinar, por su semejanza con los Amaurobiidae y con los Dictynidae. Roth (1993), para efectos de elaborar su clave, considera los géneros de Titanoecidae dentro de los Amaurobiidae. Por estas razones y por estar presente en Chile sólo una especie es que se deja fuera de la clave esta familia.

En Chile solamente Goeldia patellaris (Lehtinen 1967).

TROCHANTERIIDAE

Arañas muy particulares y curiosas por la forma deprimida del cuerpo cefalotórax y abdomen, tanto que semejan una lámina. Son de un considerable tamaño, de 15-18 mm. El cefalotórax es pardoanaranjado y el abdomen gris.

Para Chile se ha descrito sólo una especie, Doliomalus cimicoides, endémico de nuestro país (Platnick 1984, 1985, 1990).

ULOBORIDAE: (TEJEDORAS ORBICULARES CRIBELATADAS)

Constructoras de telas orbiculares o sólo con una sección triangular, en general los hilos están dispuestos horizontalmente. Una particularidad de las especies de esta familia es que carecen de glándula de veneno (Roth 1978).

En Chile tres especies: Orinomana mana, Sybota abdominalis y S. osornis (Platnick 2005).

\section{ZODARIIDAE}

Son arañas de movimiento muy rápido, viven asociadas al suelo, entre rocas o la hojarasca. Las especies del género Storena (no presente en Chile) son conocidas como cazadoras de hormigas (Watson \& Dallwitz 2004).

Para Chile se han descrito tres géneros: Cybaeodamus, Cyrioctea y Platnickia y siete especies (Platnick 2005).

\section{ZORIDAE (ARAÑAS FANTASMA)}

Estas arañas no fabrican tela para cazar sus presas, más bien las persiguen y las capturan. Frecuentemente pueden ser encontradas durante el día en la vegetación baja o en el suelo.

Solamente una especie descrita para Chile, Odo patricius (Platnick 2005).

\section{DISCUSION}

Se han descrito 55 familias para la aracnofauna chilena de un total de 110 (Platnick 2005), corres- pondiendo aproximadamente al $50 \%$ del total de familias conocidas a nivel mundial. Entre las familias con mayor número de representantes en el país están Linyphiidae y Theridiidae; y además están presentes familias como Trochanteridae con una distribución Gondwanica, con registros en Australia, cono sur de América y Africa, y numerosas especies endémicas.

Debido a que existen problemas para delimitar taxonómicamente algunas familias, o por ser monogenéricas $\mathrm{y} / \mathrm{o}$ tener sus holotipos perdidos, no se han incluido en la clave las familias Amphinectidae, Clubionidae, Desidae, Synotaxidae, Tengellidae y Titanoecidae. Por ejemplo las familias Desidae y Titanoecidae están relacionadas con los Amaurobiidae y han sido consideradas como subfamilias (Leech 1972, fide Roth 1993); sin embargo, actualmente ambos taxa son válidos, pero Roth (1993) incluye algunas especies de Titanoecidae y Desidae dentro de los Amaurobiidae para efectos de elaborar su clave y las demás especies, dentro de las familias que corresponden. Similar situación ocurre en dos géneros presentes en el país, Calcadia y Metaltella, que inicialmente estaban en las familias Pisauridae y Dictynidae respectivamente, pero Lehtinen (1967) transfirió a la familia Amaurobiidae; sin embargo, Davies (1998, fide Platnick 2005) transfiere ambos géneros a la familia Amphinectidae. Las familias Sinotaxidae y Amphinectidae tampoco fueron incluidas en la clave, por no disponer de ejemplares machos y hembras para la obtención de caracteres diagnósticos y porque en las diagnosis de las familias sólo se incluyen caracteres de los machos (Forster et al. 1990). En las familias Clubionidae y Tengellidae, los holotipos de las especies descritas para Chile se han reportado como perdidos, por lo que al no tener la seguridad que estén presentes en nuestro país se omiten de la clave dicotómica.

Las familias pertenecientes al infraorden Mygalomorphae ya han sido estudiadas por Raven (1985) y Goloboff $(1994,1995)$ y elaboran claves para su identificación, por lo que no se incluyeron en la clave propuesta.

En el caso de las especies con distribución conocida como neotropical o cosmopolita, a pesar de no haber revisado ejemplares recolectados en el país, sí se han incluido en este estudio.

Finalmente es importante destacar que la clave dicotómica representa un instrumento de utilidad para facilitar el estudio de las arañas en nuestro 
país, y que existen diversos problemas sistemáticos $\mathrm{y}$ filogenéticos que deben abordarse en un futuro próximo.

\section{AGRADECIMIENTOS}

Al Dr. Martín Ramírez por su tiempo y sugerencias. Al profesor Hugo I. Moyano quién aportó valiosos comentarios sobre el manuscrito. Este trabajo fue realizado gracias al aporte del Proyecto DIUC $\mathrm{N}^{\circ}$ 204.113.065-1.0.

\section{BIBLIOGRAFIA}

Agnarsson, I. 2004. Morphological phylogeny of cobweb spiders and their relatives (Araneae, Araneoidea, Theridiidae). Zoological Journal of the Linnean Society 141: 447 - 626.

ArCher, A. F. 1963. Catálogo de arañas chilenas de las familias de la división Metarachnae. Publicaciones Ocasionales Museo Nacional de Historia Natural de Santiago. 1: 1-32.

Artaza O., J. Fuentes \& R. Schindler. 1982. Latrodectismo: evaluación clínico-terapéutica de 89 casos. Revista Médica de Chile 110: 11011105.

Artaza O., J. Fuentes, P. Gómez \& R. Morris. 1984. Latrodectismo (II): evaluación clínico-terapéutica de 78 casos. Parasitología al Día. 8: 45-49

Barrio, A. \& A. Ibarra-Grasso. 1966. 16 casos de Loxoscelismo grave ocurridos en la ciudad de Buenos Aires y alrededores en los últimos años. Memorias Instituto de Butantan. Simposio Internacional. 33(3):809-820.

Cannals, M. M.E. Casanueva \& M. Aguilera. 2004. Cuáles son las especies de arañas peligrosas en Chile. Revista Médica de Chile 132: 773-776.

Casanueva, M. E. 1980. Los licósidos de Chile. Estudio biológico y taxonómico por los métodos de sistemática alfa y taxonómica numérica (Araneae: Lycosidae). Gayana Zoológica 42:1-76.

Cenalovic. K. 1976. Catálogo de los Arachnida: Scorpiones, Pseudoscorpiones, Opiliones, Acari, Araneae y Solifugae de la XII Región de Chile, Magallanes. Incluyendo la antártica chilena (Chile). Gayana 37: 1-108.

Coddington J. A. \& H. W. Levi. 1991. Systematics and evolution of spiders. Annual Review of Ecology Systematic 22: 562-595.

Comstock, J. H. 1975. The spider book. $4^{\text {th }}$ Ed. Cornell University Press. U.K. 729 pp.

Donoso, R. 1948. Consideraciones sobre aracnoidismo cutáneo en Chile. Apartado de los archivos uruguayos de medicina, cirugía, y especialidades. Tomo XXXIII 5-6: 184-206.
FLÓREZ, E. 1999. Estructura y composición de una comunidad de arañas (Araneae) en un bosque muy seco tropical de Colombia. Boletín Entomología Venezolana 14(1): 37-51.

Forster, R. R. \& N. I. Platnick. 1984. A review of the archaeid spiders and their relatives, with notes on the limits of the superfamily Palpimanoidea (Arachnida, Araneae). Bulletin of the American Museum of Natural History 178: 1-106.

Forster, R. R. \& N. I. Platnick. 1985. A review of the austral spider family Orsolobidae (Arachnida, Araneae), with notes on the superfamily Dysderoidea. Bulletin of the American Museum of Natural History 181: 1-230.

Forster, R. R., N. I. Platnick \& M. R. Gray. 1987. A review of the spider superfamilies Hypochiloidea and Austrochiloidea (Araneae, Araneomorphae). Bulletin of the American Museum of Natural History 185: 1-116.

Forster, R. R., N. I. Platnick \& J. Coddington. 1990. A proposal and review of the spider family Synotaxidae (Araneae, Araneoidea), with notes on theridiid interrelationships. Bulletin of the American Museum of Natural History 193: 1116.

GajARdo-ToBar, R. 1963. La clínica del aracnoidismo. Hospital de Viña del Mar Boletín trimestral. XIX:3.

Galiano, M. E. 1980. Catálogos de los especímenes típicos de Salticidae (Araneae) descritos por Cándido F. de Mello-Leitão. Primera parte. Physis, B. Aires (secc. c). 39:31-40.

Galiano, M. E. 1985. Revisión del género Hurius Simon 1901 (Araneae: Salticidae). Journal of Arachnology 13:9-18

Galiano, M. E. 1988. Revisión de los géneros del grupo Hurieae (Araneae, Salticidae). Journal of Arachnology 15: 285-301.

Gertsch, W. J. \& F. EnNiK. 1983. The spider genus Loxosceles in North America, Central America, and the West Indies (Araneae, Loxoscelidae). Bulletin of the American Museum of Natural History $175: 264-360$.

Gerschman De P., B. S. \& R. D. Schiapelli. 1979. Caracteres morfológicos válidos en la sistemática del género Sicarius (Walckenaer 1847) Araneae: Sicariidae. Acta Zoologica Lilloana 35: 87-96.

Goloboff, P. A. 1994. Migoidea de Chile, nuevas o poco conocidas (Araneae: Mygalomorphae). Revista de la Sociedad Entomológica Argentina 53: 65-74.

Goloboff, P. A. 1995. A revision of South American spiders of the family Nemesiidae (Araneae: Mygalomorphae) Part I: species from Peru, Chile, Argentina, and Uruguay. Bulletin of the American Museum of Natural History 224: 1-189.

Goloboff, P. A. \& N. I. Platnick. 1987. A review of the Chilean spiders of the Superfamily Migoidea (Araneae, Mygalomorphae). American Museum Novitate 2888: 1-15.

Gómez, P., J. González, G. Cabrera \& M. Rudolph. 1986. 
Evaluación de un nuevo tratamiento farmacológico para el latrodectismo. Parasitología al Día 10: 8-12.

Gómez, P., J. GonzÁlez, G. Cabrera \& M. Rudolph 1987. Alteraciones electrocardiográficas y de laboratorio en el latrodectismo. Parasitología al Día. 11: 8-11

Grismado, C. J. \& L. Lopardo. 2003a. A new species of Austrochilus from Chile (Araneae, Austrichilidae, Austrochilinae). Journal of Arachnology 31: 148150.

Grismado, C. J. \& L. Lopardo. 2003b. Nuevos datos sobre la distribución geográfica de las familias australes de arañas Malkaridae y Mecysmaucheniidae (Arachnida: Araneae), con la descripción de la hembra de Mecysmauchenius thayerae Forster \& Platnick. Revista Ibérica de Aracnología 8: 37-43.

GRISwOLD, C. E. 1993. Investigations into the phylogeny of the lycosoid spiders and their kin (Arachnida: Araneae: Lycosoidea). Smithsonian Contribution in Zoology 539: 1-39.

Griswold, C. E., J. A. Coddington, G. Hormiga \& N. ScharfF. 1998. Phylogeny of the orb-web building spiders (Araneae, Orbiculariae: Deinopoidea, Araneoidea). Zoological Journal of the Linnean Society. 123: 1-99.

Griswold, C. E., J. A. Coddington, N. I. Platnick \& R. R. Forster. 1999. Towards a phylogeny of entelegyne spiders (Araneae, Araneomorphae, Entelegynae). Journal of Arachnology 27: 53-63.

Huber, B. A. 2000. New world pholcid spiders (Araneae: Pholcidae): A revision at generic level. Bulletin of the American Museum of Natural History 254: 1-348.

Kaston, B. J. 1978. How to know the spiders. $3^{\text {a }}$ ed. Wm C. Brown. Dubuque, Iowa. 272 pp.

Legendre, R. \& R. CALDERÓN C. 1984. Liste systématique des aragnées mygalomorphes du Chili. Bull. Mus. Nat. Hist. Nat. Paris (4) 6 (a): 1021-1065.

Lehtinen, P. T. 1967. Classification of the cribellate spiders and some allied families, with notes on the evolution of the suborder Araneomorpha. Annales Zoologici Fennici 4: 199-468.

Levi, H. W. 1967. The Theridiid spider fauna of Chile. Bulletin of the Museum of Comparative Zoology. 136:1-20

Lopardo, L., M. J. Ramírez, C. Grismado \& L. A. CAmpagnucCI. 2004. Web Building behavior and the phylogeny of Austrochiline spiders. Journal of Arachnology 32: 42-54.

Mello-Leitão, C. F. DE. 1926. Une nouvelle espece di Coenypha. Revista Chilena de Historia Natural 30: 322-323.

Mello-Leitão, C. F. DE. 1943. Arañas do Chile colegidas pelo Dr. J. C. Carballo. Revista Brasileña de Biología 3: 403-409.

Molina, J. I. (1782) 1987. Ensayo sobre la Historia Natural de Chile. $1^{\text {a }}$ ed español. Ediciones Maule. Santiago de Chile. 384 pp.

Moulder B. C. \& D. E. Riechle. 1972. Significance of spider predation in the energy dynamics of forest- floor arthropod communities. Ecologucal Monograph 42(4): 473-498.

Nicolet, A. C. 1849. Arácnidos. En Gay, C. (ed.), Historia física y política de Chile. Zoología, 3: 319-543.

Nyffeler M., W. Sterling \& D. Dean. 1994. How spiders make a living. Environmental Entomology 23(6):1357-1367.

Platnick, N. I. 1983. A review of the chilensis group of the spider genus Echemoides (Araneae, Gnaphosidae). American Museum Novitate 2760: 1-18.

Platnick, N. I. 1984. On the male of Doliomalus (Araneae, Gnaphosoidea). Journal of Arach-nology 11: 451452.

Platnick, N. I. 1985. Studies on Malagasy spiders, 2. The family Trochanteriidae (Araneae, Gnaphosoidea), with a revision of the genus Platyoides. American Museum Novitate 2808: $1-17$.

Platnick, N. I. 1989. Advances in spider taxonomy 19811987. A supplement to Brignoli's a catalogue of the Araneae described between 1940 and 1981. Manchester University press. Manchester and New York. U.S.A. 673pp.

Platnick, N. I. 1990. Spinneret morphology and the phylogeny of ground spiders (Araneae, Gnaphosoidea). American Museum Novitate 2978: 1-42.

Platnick. N. I. 2005. The World Spider Catalog, Version 6.0. American Museum of Natural History. http:/ /research.amnh.org/entomology/spiders/cata$\log 81-87 /$ index.html

Platnick, N. I. \& A. D. Brescovit. 1995. On Unicorn, a new genus of the spider family Oonopidae (Araneae, Dysderoidea). American Museum Novitate 3152: 1-12.

Platnick, N. I. \& R. R. Forster. 1986. On Teutoniella, an American genus of the spider family Micropholcommatidae (Araneae, Palpimanoidea). American Museum Novitate 2854: 1-9.

Platnick, N. I. \& R. R. Forster. 1987. On the first American spiders of the subfamily Sternodinae (Araneae, Malkaridae). American Museum Novitate 2894: 1-12.

Platnick, N. I. \& R. R. Forster. 1989. A revision of the temperate South American and Australasian spiders of the family Anapidae (Araneae, Araneoidea). Bulletin of the American Museum of Natural History 190: 1-139.

Platnick, N. I., C. J. Grismado \& M. J. Ramírez. 1999. On the genera of the spider subfamily Otiothopinae (Araneae, Palpimanidae). American Museum Novitate 3257: 1-25.

Platnick, N. I. \& M. U. Shadab. 1983a. A revision of the American spiders of the genus Zelotes (Araneae, Gnaphosidae). Bulletin of the American Museum of Natural History 174: 97-192.

Platnick, N. I. \& M. U. Shadab. 1983b. A revision of the Neotropical spider genus Apodrassodes (Araneae, Gnaphosidae). American Museum Novitate 2763: 1-14. 1983. 
Gayana 69(2), 2005

Platnick, N. I. \& M. U. Shadab. 1993. A review of the pirate spiders (Araneae, Mimetidae) of Chile. American Museum Novitate 3074:1-30.

Porter, C.1917. Apuntes sobre aracnología chilena. Sinopsis de los Disdéridos. Revista Chilena de Historia Natural 21(6): 172-182

Porter, C.1918. Apuntes sobre aracnología chilena. El género Meta. Revista Chilena de Historia Natural 22(1): 16-19.

Porter, C.1930. Los artrópodos en la obra de Molina. Revista Chilena de Historia Natural 34: 161-163

Ramírez, M. J. 1995. Revisión y filogenia del género Monapia, con notas sobre otras Araurobioidinae (Araneae, Anyphaenidae). Boletín de la Sociedad de Concepción, Chile. 66: 71-102.

Ramírez, M. J. 1997. Revisión y filogenia de los géneros Ferrieria y Acanthoceto (Araneae: Anyphaenidae, Amaurobioidinae). Iheringia, Serie Zoológica 82:173-203.

Ramírez, M. J. 1999. Orden Araneae. En Crespo, F. A., Iglesias, M. S. \& Valverde, A. C. (ed.), El ABC en la determinación de artrópodos. Claves para especímenes presentes en la Argentina I. Editorial CCC Educando, Buenos Aires. 107 pp.

Ramírez, M. J. 2003. The spider subfamily Amaurobioidinae (Araneae: Anyphaenidae): A phylogenetic revision at the generic level. Bulletin of the American Museum of Natural History 277: 1-262.

Ramírez, M. J., A. B. Bonaldo \& A.D. Brescovit. 1997. Revisión del género Macerio y comentarios sobre la ubicación de Cheiracanthium, Tecution y Helebiona (Araneae, Miturgidae, Eutichurinae). Iheringia, Serie Zoológica 82:43-66.

Ramírez, M. J., C. Grismado \& T. Blick. 2004. Notes of the spider family Agelenidae in southern South America (Arácnida: Araneae). Revista Ibérica de Aracnología 9: 179-182.

Ramírez, M. J., L. Lopardo \& A. B. Bonaldo. 2001. A review of the Chilean spider genus Olbus, With notes on the relationships of the Corinnidae (Arachnida, Araneae). Insect Systematic Evolution 31:4; 441-462.

Ramírez, M. J., L. Lopardo \& N. I. Platnick. 2004. Notes on Chilean anapids and their webs. American Museum Novitate 3428: 1-13.

RAVEN, R. J. 1985. The spider infraorder Mygalomorphae (Araneae): Cladistics and systematics. Bulletin of the American Museum of Natural History 182: $1-180$

Roth, V. D. 1993. Spider genera of North America. $3^{\text {rd }}$ Ed. Arizona, U.S.A. 203 pp.
SChenone, H. 1959. Aspectos prácticos en la clínica del síndrome del latrodectismo y su tratamiento con Neostigmina (Prostigmina). Boletín Chileno de Parasitología 14: 80-82

Schenone, H. \& T. Lentoja. 1975. Notas sobre la biología y distribución geográfica de las arañas del género Loxosceles. Boletín Chileno de Parasitología 30:27-29.

Schenone, H., S. Rubio, F. Villarroel \& A. Rojas. 1975. Epidemiología y curso clínico del Loxoscelismo, estudio de 133 casos causados por la mordedura de la araña de los rincones (Loxosceles laeta). Boletín Chileno de Parasitología 30: 6-17.

Simon, E. 1895. Histoire naturelle des araignées. Paris, 1: 761-1084.

Tullgren, A. 1901. Contribution to the knowledge of the spider fauna of the Magellan Territories. Svenska Expeditionen till Magellansländerna 2(10): 181263.

Watson, L. \& M. J. Dallwitz. 2004. The Families of Spiders Represented in the British Isles, http:// delta-intkey.com/britsp/index.htm

Wolff, R. J. 1977. The cribellate genus Tengella (Araneae: Tengellidae?). Journal of Arachnology 5: $139-144$

Zapfe, H. 1955. Filogenia y función en Austrochilus manni Gertsch y Zapfe (Araneae-Hypochilidae). Trabajos del Laboratorio de Zoología de la Universidad de Chile 2: 1-53.

ZAPFE H. 1959. Clave para determinar familias y géneros de arañas chilenas. Investigaciones Zoológicas Chilenas V:149-188.

ZaPfe H. 1961a. Distribución ecológica de Araneae en la Quebrada de La Plata, La Rinconada, Maipú. Investigaciones Zoológicas Chilenas VII: 125-128.

ZaPfe H. 1961b. Distribución altitudinal de Araneae en el valle del Río Mapocho. Investigaciones Zoológicas Chilenas VII: 128-132.

ZAPFE H. 1961c. Biogeografía de las arañas en Chile. Investigaciones Zoológicas Chilenas. Vol VII: 133-136.

ZAPFE H. 1961d. Arañas tropicales en nuestro país. Investigaciones Zoológicas Chilenas VII:137-140.

ZAPFE H. 1961e. La familia Palpimanidae en Chile. Investigaciones Zoológicas Chilenas VII:141-144.

ZAPFE H. 1961f. La familia Filistatidae en Chile. Investigaciones Zoológicas Chilenas VII:145-150.

ZapfE H. 1961g. La familia Migidae en Chile. Investigaciones Zoológicas Chilenas VII:151-158.

Zapfe-Mann, H. 1979. Pardosa anfibia, nueva especie (Lycosidae: Araneae) Noticiario mensual Museo Nacional de Historia Natural 272:3-7. 